\title{
Deformation-Based Surface Morphometry Applied to Gray Matter Deformation
}

\author{
Moo K. Chung ${ }^{1,2}$, Keith J. Worsley ${ }^{3,4}$, Steve Robbins ${ }^{4}$, Tomas Paus ${ }^{4}$, \\ Jonathan Taylor ${ }^{5}$, Jay N. Giedd ${ }^{6}$, Judith L. Rapoport ${ }^{6}$, Alan C. Evans ${ }^{4}$ \\ ${ }^{1}$ Department of Statistics \\ ${ }^{2}$ W.M. Keck Laboratory for Functional Brain Imaging \& Behavior \\ University of Wisconsin, Madison, Wisconsin 53706 \\ ${ }^{3}$ Department of Mathematics and Statistics \\ ${ }^{4}$ Montreal Neurological Institute, McGill University, Canada \\ ${ }^{5}$ Department of Statistics, Stanford University \\ ${ }^{6}$ Child Psychiatry Branch, National Institute of Mental Health, \\ NIH, Bethesda, Maryland 20892
}

August 15, 2002

\section{Corresponding Address:}

Moo K. Chung

Department of Statistics

1210 West Dayton Street

University of Wisconsin-Madison

Madison, WI 53706-1685

Phone: (608)262-1287, Fax: (608)262-0032

E-mail: mchung@stat.wisc.edu 


\begin{abstract}
We present a unified statistical approach to deformation-based morphometry applied to the cortical surface. The cerebral cortex has the topology of a 2D highly convoluted sheet. As the brain develops over time, the cortical surface area, thickness, curvature and total gray matter volume change. It is highly likely that such age-related surface changes are not uniform. By measuring how such surface metrics change over time, the regions of the most rapid structural changes can be localized. We avoided using surface flattening, which distorts the inherent geometry of the cortex in our analysis and it is only used in visualization. To increase the signal to noise ratio, diffusion smoothing, which generalizes Gaussian kernel smoothing to an arbitrary curved cortical surface, has been developed and applied to surface data. Afterwards, statistical inference on the cortical surface will be performed via random fields theory. As an illustration, we demonstrate how this new surface-based morphometry can be applied in localizing the cortical regions of the gray matter tissue growth and loss in the brain images longitudinally collected in the group of children and adolescents.
\end{abstract}

Keywords: Cerebral Cortex, Cortical Surface, Brain Development, Cortical Thickness, Brain Growth, Brain Atrophy, Deformation, Morphometry 


\section{Introduction}

The cerebral cortex has the topology of a 2-dimensional convoluted sheet. Most of the features that distinguish these cortical regions can only be measured relative to that local orientation of the cortical surface (Dale and Fischl, 1999). As brain develops over time, cortical surface area as well as cortical thickness and the curvature of the cortical surface change. As shown in the previous normal brain development studies, the growth pattern in developing normal children is nonuniform over whole brain volume (Chung et al., 2001; Giedd et al., 1999; Paus et al., 1999, Thompson et al., 2000). Between ages 12 and 16, the corpus callosum and the temporal and parietal lobes shows the most rapid brain tissue growth and some tissue degeneration in the subcortical regions of the left hemisphere (Chung et al. 2001, Thompson et al., 2000). It is equally likely that such age-related changes with respect to the cortical surface are not uniform as well. By measuring how geometric metrics such as the cortical thickness, curvature and local surface area change over time, any statistically significant brain tissue growth or loss in the cortex can be detected.

The first obstacle in developing surfaced-based morphometry is the automatic segmentation or extraction of the cortical surfaces from MRI. It requires first correcting RF inhomogeneity artifacts. We have used nonparametric nonuniform intensity normalization method (N3), which elimates the dependence of the field estimate on anatomy (Sled et al., 1998). The next step is the tissue classification into three types: gray matter, white matter and cerebrospinal fluid (CSF). An artificial neural network classifier (Ozkan et al., 1993; Vaken, 1996) or a mixture model cluster analysis (Good et al., 2001) can be used to segment the tissue types automatically. After the tissue classification, the cortical surface is usually generated as a smooth triangular mesh. The most widely used method for triangulating the surface is the marching cubes algorithm (Lorensen and Cline, 1987). Level set method (Sethian, 1996) or deformable surfaces method (Davatzikos, 1995) are also available. In our study, we have used the anatomic segmentation using proximities (ASP) method (MacDonald et al., 2000), which is a variant of the deformable surfaces method, to generate cortical triangular meshes that has the topology of a sphere. Brain substructures such as the brain stem and the cerebellum were removed. Then an ellipsoidal mesh that already had the topology of 
a sphere was deformed to fit the shape of the cortex guranting the same topology. The resulting triangular mesh will consist of 40,962 vertices and 81,920 triangles with the average internodal distance of $3 \mathrm{~mm}$. Partial voluming is a problem with the tissue classifier but topology constraints used in ASP method were shown to provide some correction by incorporating many neuroanatomical a priori information (MacDonald, 1997; MacDonald et al., 2000). The triangular meshes are not constrained to lie on voxel boundaries. Instead the triangular meshes can cut through a voxel, which can be considered as correcting where the true boundary ought to be. Once we have a triangular mesh as the realization of the cortical surface, we can model how the cortical surface deforms over time.

In modeling the surface deformation, a proper mathematical framework might be found in both differential geometry and fluid dynamics. The concept of the evolution of phaseboundary in fluid dynamics (Drew, 1991; Gurtin and McFadden, 1991), which describes the geometric properties of the evolution of boundary layer between two different materials due to internal growth or external force, can be used to derive the mathematical formula for how the surface change. It is natural to assume the cortical surfaces to be a smooth 2-dimensional Riemannian manifold parameterized by $u^{1}$ and $u^{2}$ :

$$
\mathbf{X}\left(u^{1}, u^{2}\right)=\left\{x_{1}\left(u^{1}, u^{2}\right), x_{2}\left(u^{1}, u^{2}\right), x_{3}\left(u^{1}, u^{2}\right):\left(u^{1}, u^{2}\right) \in D \subset \mathbb{R}^{2}\right\} .
$$

A more precise definition of a Riemannian manifold and a parameterized surface can be found in Boothby (1986), Carmo (1992) and Kreyszig (1959). If $D$ is a unit square in $\mathbb{R}^{2}$ and a surface is topologically equivalent to a sphere then at least two different global parameterizations are required. Surface parameterization of the cortical surface bas been done previously by Thompson and Toga (1996) and Joshi et al. (1995). From the surface parameterization, Gaussian and mean curvatures of the brain surface can be computed and used to characterize its shape (Dale and Fischl, 1999; Griffin, 1994; Joshi et al., 1995). In particular, S.C. Joshi et al. (1995) used the quadratic surface in estimating the Gaussian and mean curvature of the cortical surfaces.

By combining the mathematical framework of the evolution of phase-boundary with the statistical framework developed for 3D whole brain volume deformation (Chung et al., 2001), anatomical variations associated with the deformation of the cortical surface can be 


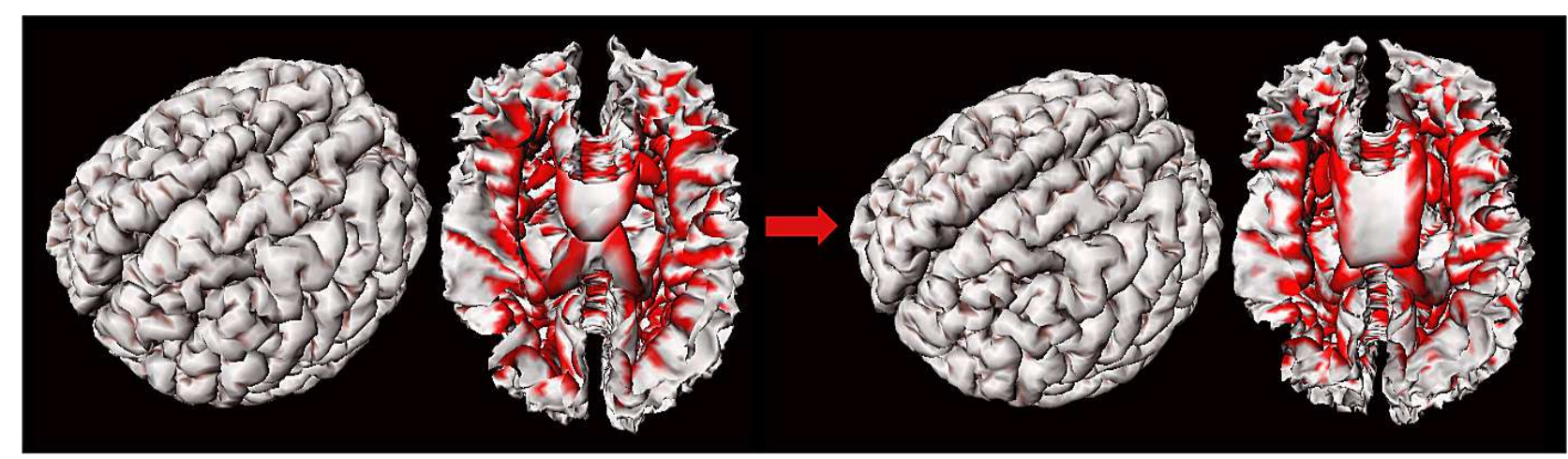

Figure 1: The outer and the inner cortical surfaces of a single subject at age 14 (left) and at age 19 (right) showing globally similar cortical patterns. The top of the inner cortical surface has been removed to show predominant ventricle enlargement. The red color is the region where the mean curvature is greater than 0.01 .

statistically quantified. Using the same stochastic assumption on the deformation field used in Chung et al. (2001), we will localize the region of brain shape changes based on three surface metrics: area dilatation rate, cortical thickness and curvature changes and show how these metrics can be used to characterize the brain surface shape changes over time.

As an illustration of our unified approach to deformation-based surface morphometry, we will demonstrate how the surface-based statistical analysis can be applied in localizing the cortical regions of tissue growth and loss in brain images longitudinally collected in a group of children and adolescents.

\section{Modeling Surface Deformation}

Let $\mathbf{U}(\mathbf{x}, t)=\left(U_{1}, U_{2}, U_{3}\right)^{t}$ be the $3 \mathrm{D}$ displacement vector required to deform the structure at $\mathbf{x}=\left(x_{1}, x_{2}, x_{3}\right)$ in gray matter $\Omega_{0}$ to the homologous structure after time $t$. Whole gray matter volume $\Omega_{0}$ will deform continuously and smoothly to $\Omega_{t}$ via the deformation $\mathbf{x} \rightarrow \mathbf{x}+\mathbf{U}$ while the cortical boundary $\partial \Omega_{0}$ will deform to $\partial \Omega_{t}$. The cortical surface $\partial \Omega_{t}$ may be considered as consisting of two parts: the outer cortical surface $\partial \Omega_{t}^{\text {out }}$ between the gray matter and CSF and the inner cortical surface $\partial \Omega_{t}^{i n}$ between the gray and white matter (Figure 2), i.e.

$$
\partial \Omega_{t}=\partial \Omega_{t}^{\text {out }} \cup \partial \Omega_{t}^{\text {in }}
$$




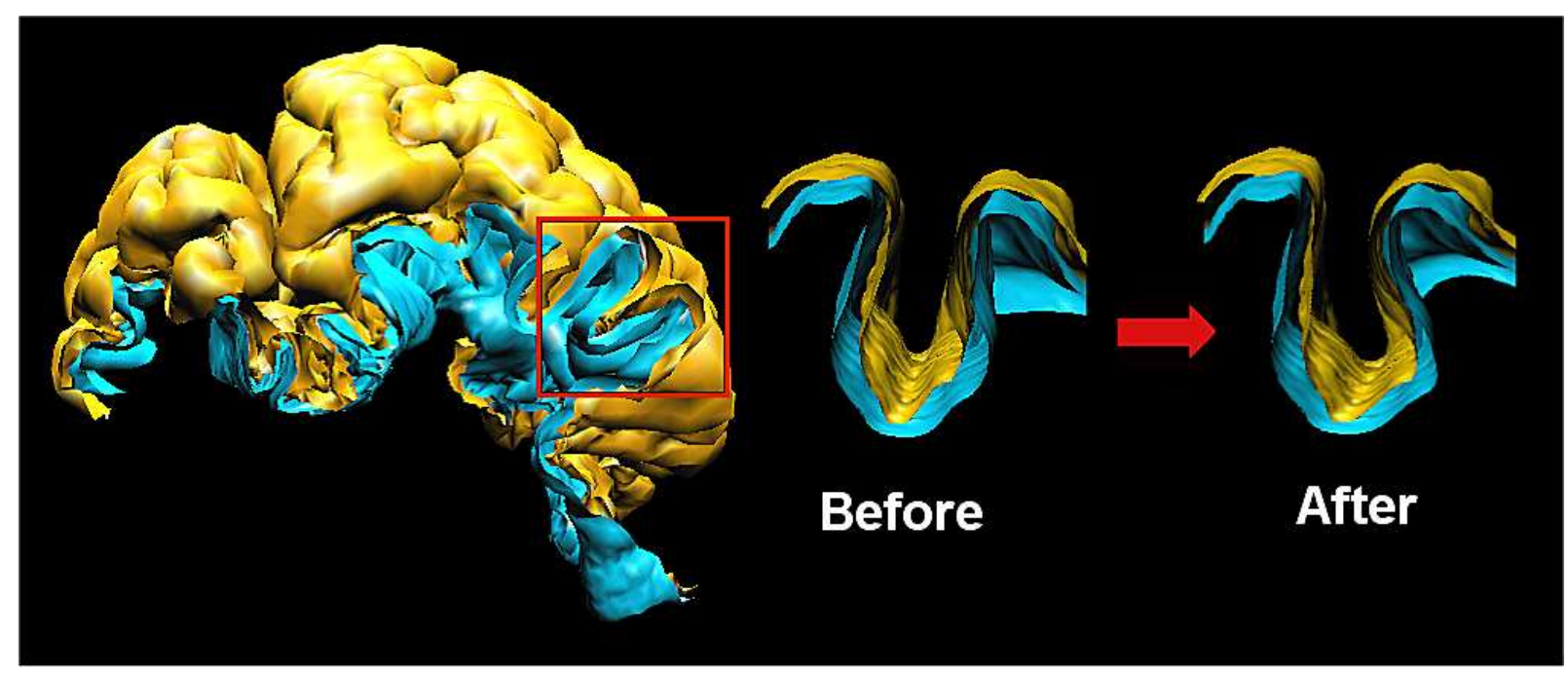

Figure 2: Yellow: outer cortical surface, blue: inner cortical surface. Gray matter deformation causes the geometry of the both outer and inner cortical surfaces to change. The deformation of the surfaces can be written as $\mathbf{x} \rightarrow \mathbf{x}+\mathbf{U}(\mathbf{x}, t)$, where $\mathbf{U}$ is the surface displacement vector field.

Although we will exclusively deal with the deformation of the cortical surfaces, our deformationbased surface morphometry can be equally applicable to the boundary of any brain substructure.

We propose the following stochastic model on the displacement velocity $\mathbf{V}=\partial \mathbf{U} / \partial t$, which has been used in the analysis of whole brain volume deformation (Chung et al., 2001):

$$
\mathbf{V}(\mathbf{x})=\mu(\mathbf{x})+\Sigma^{1 / 2}(\mathbf{x}) \epsilon(\mathbf{x}), \mathbf{x} \in \Omega_{0}
$$

where $\mu$ is the mean displacement velocity and $\Sigma^{1 / 2}$ is the $3 \times 3$ symmetric positive definite covariance matrix, which allows for correlations between components of the displacement fields. The components of the error vector $\epsilon$ are are assumed to be independent and identically distributed as smooth stationary Gaussian random fields with zero mean and unit variance. It can be shown that the normal component of the displacement velocity $\mathbf{V}=\partial \mathbf{U} / \partial t$ restricted on the boundary $\partial \Omega_{0}$ uniquely determine the evolution of the cortical surface (Chung et al., 2002).

Estimating the surface displacement fields $\mathbf{U}$ and the surface extraction can be performed at the same time by the ASP algorithm. First, an ellipsoidal mesh placed outside the brain was shrunk down to the surface $\partial \Omega_{0}^{i n}$. The vertices of the resulting inner mesh are indexed and 


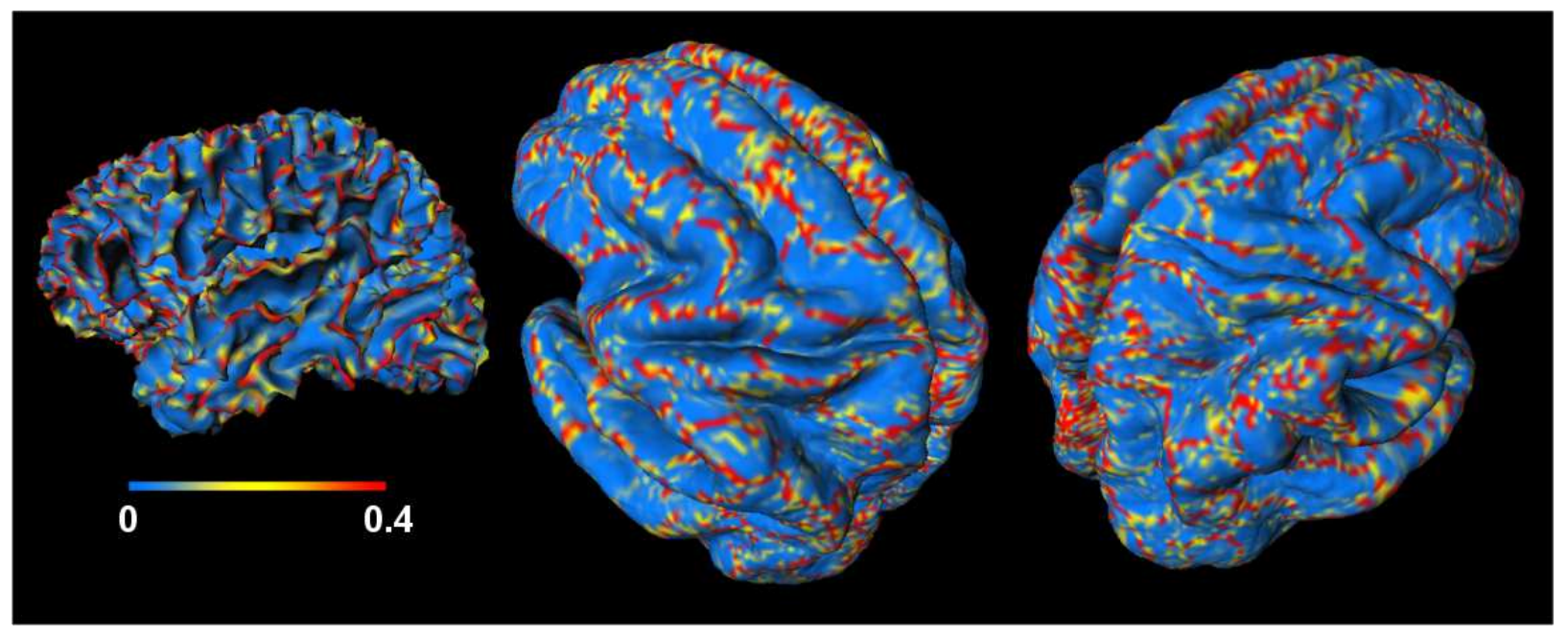

Figure 3: Individual gyral patterns mapped onto the surface atlas $\partial \Omega_{\text {atlas }}$. The gyral patterns (yellow and red lines) are extracted by computing the bending metric on the inner cortical surface (left). The middle and right figures show the mapping of the gyral pattern of a single subject (left) onto the atlas surface. The gyri of the subject matche the gyri of the atlas illustrating a close homology between the surface of an individual subject and surface atlas. If there is no homology between the corresponding vertices, we would have complete misalignment .

the ASP algorithm will deform the inner mesh to fit the outer surface $\partial \Omega_{0}^{\text {out }}$. by minimizing a cost function that involves bending, stretch and other topological constraints (MacDonald et al., 2000). The vertices indexed identically on both meshes will lie within a very close proximity and these define the automatic linkage in the ASP algorithm. To generate the outer surface $\partial \Omega_{t}^{\text {out }}$ at later time $t$, we start with the inner surface $\partial \Omega_{0}^{\text {in }}$, and then deform it to match the outer surface $\partial \Omega_{t}^{\text {out }}$ by minimizing the same cost function. Starting with the same mesh in two outer surface extractions, each point on $\partial \Omega_{0}^{i n}$ gets mapped to corresponding points on $\partial \Omega_{0}^{\text {out }}$ and $\partial \Omega_{t}^{\text {out }}$. This method assumes that the shape of the cortical surface does not appreciably change within subject. This assumption is valid in the case of brain development for a short period of time as illustrated in Figure 1, where the global sulcal geometry remains stable for five year interval, although local cortical geometry shows slight changes. As reported in Chung et al. (2001), the displacement is less than $1 \mathrm{~mm} / \mathrm{year}$ in average for the same data set while the average internodal distance in triangular meshes we are using is $3 \mathrm{~mm}$. So the displacements are relatively small compared to the size of mesh itself. 
Constructing surface atlas $\partial \Omega_{\text {atlas }}$, where the statistical parametric maps (SPM) of surface metrics will be formed, is done by averaging the coordinates of corresponding vertices that have the same indices. This atlas construction method has been first introduced by MacDonald et al. (2000), where it is used to create the cortical thickness map for 150 normal subjects. The geometrical constraints such as stretch and bending terms in ASP algorithm enforces a relatively consistent correspondence on the cortical surface. Figure 3 shows the mapping of gyral pattern (red and yellow lines) of a single subject onto the atlas surface. The gyri of the subject matche the gyri of the atlas. Note the full anatomical details still presented in $\partial \Omega_{\text {atlas }}$ even after the vertex averaging. Major sulci such as the central sulcus and superior temporal sulcus are clearly identifiable. If there is no homology between corresponding vertices, one would only expect to see featureless dispersion of points.

\section{Surface Parameterization}

The ASP method generates triangular meshes consisting of 81,920 triangles evenly distributed in size. In order to quantify the shape change of the cortical surface, surface parameterization is an essential part. We model the cortical surface as a smooth 2D Riemannian manifold parameterized by two parameters $u^{1}$ and $u^{2}$ such that any point $\mathbf{x} \in \partial \Omega_{0}$ can be uniquely represented as

$$
\mathbf{x}=\mathbf{X}\left(u^{1}, u^{2}\right)
$$

for some parameter space $\mathbf{u}=\left(u^{1}, u^{2}\right) \in D \subset \mathbb{R}^{2}$. We will try to avoid global parameterization such as tensor B-splines, which are computationally expensive compared to a local surface parameterization. Instead, a quadratic polynomial

$$
z\left(u^{1}, u^{2}\right)=\beta_{1} u^{1}+\beta_{2} u^{2}+\beta_{3}\left(u^{1}\right)^{2}+\beta_{4} u^{1} u^{2}+\beta_{5}\left(u^{2}\right)^{2}
$$

will be used as a local parameterization fitted via least-squares estimation. Using the leastsquares method, these coefficients $\beta_{i}$ can be estimated. The numerical implementation can be found in Chung (2001). Slightly different quadratic surface parameterizations are also used in estimating curvatures of a macaque monkey brain surface (Joshi et al., 1995; Khaneja 
et al., 1998). Once $\beta_{i}$ are estimated, $\mathbf{X}\left(u^{1}, u^{2}\right)=\left(u^{1}, u^{2}, z\left(u^{1}, u^{2}\right)\right)^{t}$ becomes a local surface parameterization of choice.

\section{Surface-Based Morphological Measures}

As in the case of local volume change in the whole brain volume (Chung et al., 2001), the rate of cortical surface area expansion or reduction may not be uniform across the cortical surface. Extending the idea of volume dilatation, we introduce a new concept of surface area, curvature, cortical thickness dilatation and their rate of change over time.

Suppose that the cortical surface $\partial \Omega_{t}$ at time $t$ can be parameterized by the parameters $\mathbf{u}=\left(u^{1}, u^{2}\right)$ such that any point $\mathbf{x} \in \partial \Omega_{t}$ can be written as $\mathbf{x}=\mathbf{X}(\mathbf{u}, t)$. Let $\mathbf{X}_{i}=\partial \mathbf{X} /$ $\partial u^{i}$ be a partial derivative vector. The Riemannian metric tensor $g_{i j}$ is given by the inner product between two vectors $\mathbf{X}_{i}$ and $\mathbf{X}_{j}$, i.e. $g_{i j}(t)=\left\langle\mathbf{X}_{i}, \mathbf{X}_{j}\right\rangle$. The Riemannian metric tensor $g_{i j}$ measures the amount of the deviation of the cortical surface from a flat Euclidean plane. The Riemannian metric tensor enables us to measure lengths, angles and areas in the cortical surface. Let $g=\left(g_{i j}\right)$ be a $2 \times 2$ matrix of metric tensors. From Chung et al, (2002), the rate of metric tensor change is approximately

$$
\frac{\partial g}{\partial t} \approx 2(\nabla \mathbf{X})^{t}(\nabla \mathbf{V}) \nabla \mathbf{X}
$$

where $\mathbf{V}=\partial \mathbf{U} / \partial t$ and $\nabla \mathbf{X}=\left(\mathbf{X}_{1}, \mathbf{X}_{2}\right)$ is a $3 \times 2$ gradient matrix. We are not directly interested in the metric tensor change itself but rather functions of $g$ and $\partial g / \partial t$, which will be used to measure surface area and curvature change.

\subsection{Local Surface Area Change}

The total surface area of the cortex $\partial \Omega_{t}$ is given by

$$
\left\|\partial \Omega_{t}\right\|=\int_{D} \sqrt{\operatorname{det} g} d \mathbf{u}
$$

where $D=X^{-1}\left(\partial \Omega_{t}\right)$ is the parameter space (Kreyszig, 1959). The term $\sqrt{\operatorname{det} g}$ is called the infinitesimal surface area element and it measures the transformed area of the unit square in the parameter space $D$ via the transformation $X(\cdot, t): D \rightarrow \partial \Omega_{t}$. The infinitesimal surface 
area element can be considered as a generalization of Jacobian, which has been used in measuring local volume in whole brain volume (Chung et al., 2001). The local surface area dilatation rate $\Lambda_{\text {area }}$ or the rate of local surface area change per unit surface area is then defined as

$$
\Lambda_{\text {area }}=\frac{1}{\sqrt{\operatorname{det} g}} \frac{\partial \sqrt{\operatorname{det} g}}{\partial t},
$$

which can be further simplified as $\Lambda_{\text {area }}=\partial(\ln \sqrt{\operatorname{det} g}) / \partial t$. If the whole gray matter $\Omega_{t}$ is parameterized by $3 \mathrm{D}$ curvilinear coordinates $\mathbf{u}=\left(u^{1}, u^{2}, u^{3}\right)$, then the dilatation rate $\partial(\ln \sqrt{\operatorname{det} g}) / \partial t$ becomes the local volume dilatation rate $\Lambda_{\text {volume }}$ first introduced in deformationbased morphometry (Chung et al., 2001). Therefore, the concepts of local area dilatation and volume dilatation rates are essentially equivalent.

In our study, two MR scans were collected for each subject at different times. Let $t_{1}^{j}$ and $t_{2}^{j}$ to be the times scans were taken for subject $j$. Then the local surface area dilatation rate $\Lambda_{\text {area }}^{j}$ for subject $j$ is estimated as a finite difference:

$$
\Lambda_{\text {area }}^{j}=\frac{\sqrt{\operatorname{det} g\left(t_{2}^{j}\right)}-\sqrt{\operatorname{det} g\left(t_{1}^{j}\right)}}{\left(t_{2}^{j}-t_{1}^{j}\right) \sqrt{\operatorname{det} g\left(t_{1}^{j}\right)}},
$$

where $g(t)$ is the matrix evaluated at $t$. Other dilatation rates that will be introduced later can be estimated in a similar fashion.

Instead of using metric tensors $g_{i j}$, it is possible to formulate local surface area change in terms of the areas of the corresponding triangles. However, this formulation assign surface area change values to each face instead of each vertex and this might cause a problem in both surface-based smoothing and statistical analysis, where values are defined on vertices. Defining scalar values on vertices from face values can be done by the weighted average of face values, which should converge to (4). It is not hard to develop surface-based smoothing and statistical analysis on values defined on faces but the cortical thickness and the curvature metric will be defined on vertices so we will end up with two separate approaches: one for metrics defined on vertices and the other for metrics defined on faces. Therefore, our metric tensor approach seems to provide a basis of unifying surface metric computations, surface-based smoothing and statistical analysis together. 
Under the assumption of stochastic model (1), the area dilatation rate can be approximately distributed as Gaussian:

$$
\Lambda_{\text {area }}(\mathbf{x})=\lambda_{\text {area }}(\mathbf{x})+\epsilon_{\text {area }}(\mathbf{x})
$$

where $\lambda_{\text {area }}=\operatorname{tr}\left[g^{-1}(\nabla X)^{t}(\nabla \mu) \nabla X\right]$ is the mean area dilatation rate and $\epsilon_{\text {area }}$ is a mean zero Gaussian random field defined on the cortical surface (Chung et al., 2002). The area dilatation rate is invariant under parameterization, i.e. the area dilatation rate will always be the same no matter which parametrization is chosen. $\lambda_{\text {area }}$ can be estimated by the sample mean $\bar{\Lambda}_{\text {area }}=\left(\sum_{j=1}^{n} \Lambda_{\text {area }}^{j}\right) / n$ and the significance of the mean will be tested via $T$ statistic.

So far our statistical modeling is centered on localizing regions of rapid morphological changes on the cortical surface but both local and global morphological measures are important in the characterization of brain deformation. Global morphometry is relatively easy compared to local morphometry with respect to modeling and computation. The total surface area $\left\|\partial \Omega_{t}\right\|$ can be estimated by the sum of the areas of 81,920 triangles generated by the ASP algorithm. Then we define the total surface area dilatation rate as

$$
\Lambda_{\text {total area }}=\frac{\partial}{\partial t} \ln \left\|\partial \Omega_{t}\right\|
$$

It can be shown that, under assumption (1), the total surface area dilatation rate $\Lambda_{\text {total area }}$ is distributed as a Gaussian random variable and hence a statistical inference on total surface area change will be based on a simple $t$ test (Chung et al., 2002). This measure will be used in determining the rate of the total surface area decreases in both outer and inner cortical surfaces between ages 12 and 16 .

\subsection{Local Gray Matter Volume Change}

Local volume dilatation rate $\Lambda_{\text {volume }}$ for whole brain volume is defined in Chung et al. (2001) using the Jacobian of deformation $\mathbf{x} \rightarrow \mathbf{x}+\mathbf{U}(\mathbf{x})$ as

$$
\Lambda_{\text {volume }}=\operatorname{tr}(\nabla \mathbf{V})=\frac{\partial}{\partial t} \operatorname{tr}(\nabla \mathbf{U})
$$

and used successfully in detecting the regions of brain tissue growth and loss in the whole brain volume. Compared to the local surface area change metric, the local volume change 

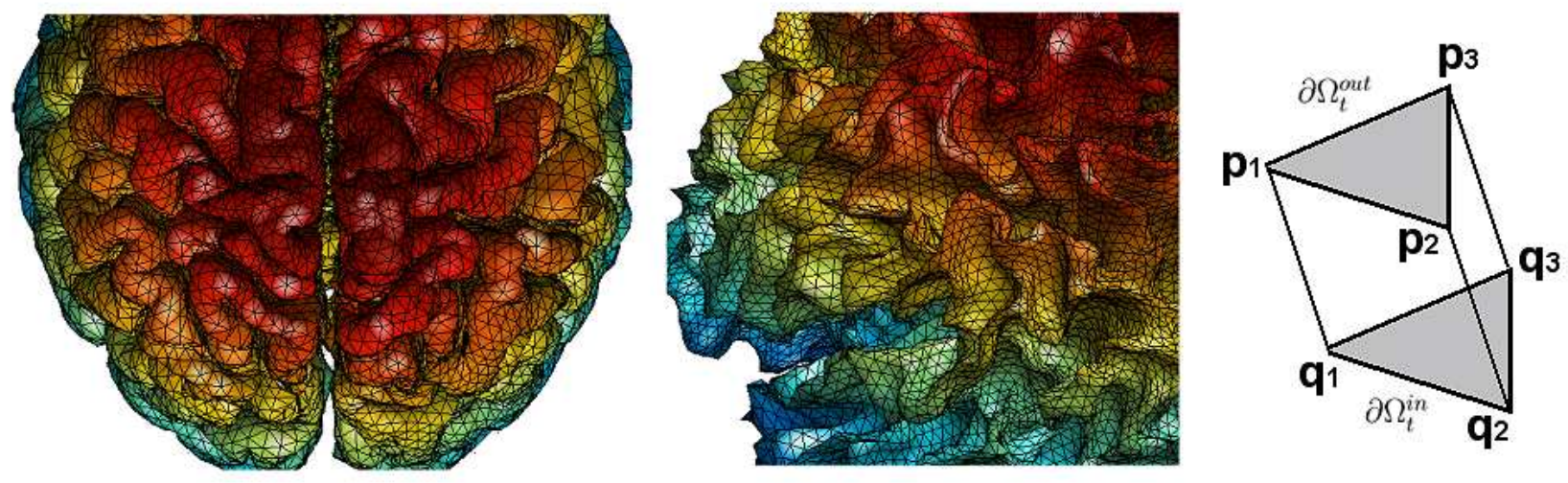

Figure 4: Outer (left) and inner (middle) triangular meshes. Triangle $\left(\mathbf{p}_{1}, \mathbf{p}_{2}, \mathbf{p}_{3}\right) \in \partial \Omega_{t}^{\text {out }}$ on the outer surface will have corresponding triangle $\left(\mathbf{q}_{1}, \mathbf{q}_{2}, \mathbf{q}_{3}\right) \in \partial \Omega_{t}^{\text {in }}$ on the inner surface. A convex-hull from 6 points $\left\{\mathbf{p}_{1}, \mathbf{p}_{2}, \mathbf{p}_{3}, \mathbf{q}_{1}, \mathbf{q}_{2}, \mathbf{q}_{3}\right\}$ will then form a triangular prism and the collection of 81,920 triangular prisms becomes the whole gray matter.

measurement is more sensitive to small deformation. If a unit cube increases its sides by one, the surface area will increase by $2^{2}-1=3$ while the volume will increase by $2^{3}-1=7$. Therefore, the statistical analysis based on the local volume change will be at least twice more sensitive compared to that of the local surface area change. So the local volume change should be able to pick out gray matter tissue growth pattern even when the local surface area change may not. In result section, the highly sensitive aspect of local volume change in relation to local surface area change will be demonstrated.

The gray matter $\Omega_{t}$ can be considered as a thin shell bounded by two surfaces $\partial \Omega_{t}^{\text {out }}$ and $\partial \Omega_{t}^{i n}$ with varying cortical thickness. In triangular meshes generated by the ASP algorithm, each of 81,920 triangles on the outer surface has a corresponding triangle on the inner surface (Figure 4). Let $\mathbf{p}_{1}, \mathbf{p}_{2}, \mathbf{p}_{3}$ be the three vertices of a triangle on the outer surface and $\mathbf{q}_{1}, \mathbf{q}_{2}, \mathbf{q}_{3}$ be the corresponding three vertices on the inner surface such that $\mathbf{p}_{i}$ is linked to $\mathbf{q}_{i}$ by ASP algorithm. The triangular prism consists of three tetrahedra with vertices $\left\{\mathbf{p}_{1}, \mathbf{p}_{2}, \mathbf{p}_{3}, \mathbf{q}_{1}\right\}$, $\left\{\mathbf{p}_{2}, \mathbf{p}_{3}, \mathbf{q}_{1}, \mathbf{q}_{2}\right\}$ and $\left\{\mathbf{p}_{3}, \mathbf{q}_{1}, \mathbf{q}_{2}, \mathbf{q}_{3}\right\}$. Then the volume of the triangular prism is given by the sum of the determinants

$$
D\left(\mathbf{p}_{1}, \mathbf{p}_{2}, \mathbf{p}_{3}, \mathbf{q}_{1}\right)+D\left(\mathbf{p}_{2}, \mathbf{p}_{3}, \mathbf{q}_{1}, \mathbf{q}_{2}\right)+D\left(\mathbf{p}_{3}, \mathbf{q}_{1}, \mathbf{q}_{2}, \mathbf{q}_{3}\right),
$$

where $D(\mathbf{a}, \mathbf{b}, \mathbf{c}, \mathbf{d})=|\operatorname{det}(\mathbf{a}-\mathbf{d}, \mathbf{b}-\mathbf{d}, \mathbf{c}-\mathbf{d})| / 6$ is the volume of a tetrahedron whose vertices are $\{\mathbf{a}, \mathbf{b}, \mathbf{c}, \mathbf{d}\}$. Afterwards, the total gray matter volume $\left\|\Omega_{t}\right\|$ can be estimated 


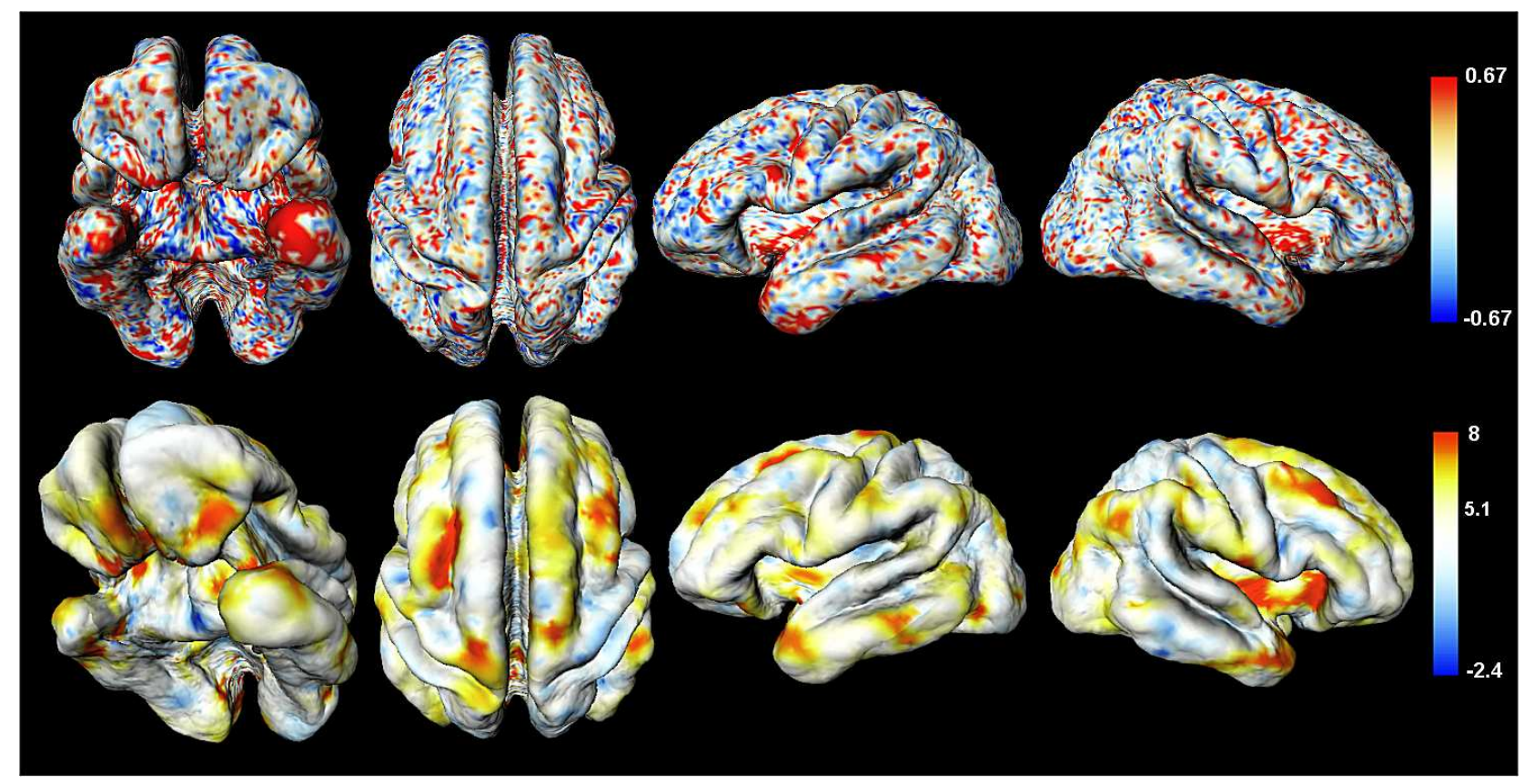

Figure 5: Top: Cortical thickness dilatation rate for a single subject mapped onto an atlas. The red (blue) regions show more than $67 \%$ thickness increase (decrease). Note the large variations across the cortex. Due to such large variations, surface-based smoothing is required to increase the signal-to-noise ratio. Bottom: $t$ statistical map thresholded at the corrected $P$ value of 0.05 ( $t$ value of 5.1). Both yellow and red regions are statistically significant regions of thickness increase. There is no region of statistically significant cortical thinning detected. The blue region shows very small $t$ value of -2.4 , which is not statistically significant.

by summing the volumes of all 81,920 triangular prisms. Similar to the total surface area dilatation rate, we define the total gray matter volume dilatation rate as

$$
\Lambda_{\text {total volume }}=\frac{\partial}{\partial t} \ln \left\|\Omega_{t}\right\|
$$

\subsection{Cortical Thickness Change}

The average cortical thickness for each individual is about 3mm (Henery and Mayhew, 1989). Cortical thickness usually varies from $1 \mathrm{~mm}$ to $4 \mathrm{~mm}$ depending on the location of the cortex. In normal brain development, it is highly likely that the change of cortical thickness may not be uniform across the cortex. We will show how to localize the cortical regions of statistically significant thickness change in brain development. Our approach introduced here can also be applied to measuring the rate of cortical thinning, possibly associated with Alzheimer's disease. As in the case of the surface area dilatation, we introduce the concept of the cortical 
thickness dilatation, which measures cortical thickness change per unit thickness and unit time. There are many different computational approaches to measuring cortical thickness but we will use the Euclidean distance $d(\mathbf{x})$ from a point $\mathbf{x}$ on the outer surface $\partial \Omega_{t}^{\text {out }}$ to the corresponding point $\mathbf{y}$ on the inner surface $\partial \Omega_{t}^{i n}$, as defined by the automatic linkages used in the ASP algorithm (MacDonald et al., 2000). The vertices on the inner triangular mesh are indexed and the ASP algorithm can deform the inner mesh to fit the outer surface by minimizing a cost function that involves bending, stretch and other topological constraints. Therefore, both the outer and the inner surfaces should match sulci to sulci and gyri to gyri, and the vertices indexed identically on both surfaces would lie within a very close proximity. One advantage of the cortical thickness metric based on this automatic linkage is that it is less sensitive to fluctuations in surface normals and regions of high curvature (MacDonald et al., 2000). A validation study for the assessment of the accuracy of the cortical thickness measure based on the ASP algorithm has been performed and found to be valid for the most of the cortex (Kanani et al., 2000). There is also an alternate method for automatically measuring cortical thickness based on the Laplace equation (Jones et al., 2000).

Let $d(\mathbf{x})=\|\mathbf{x}-\mathbf{y}\|$ be the cortical thickness computed as usual Euclidean distance between $\mathbf{x} \in \partial \Omega^{\text {out }}$ and $\mathbf{y} \in \partial \Omega^{\text {in }}$. We define the cortical thickness dilatation rate as the rate of the change of the thickness per unit thickness and unit time, i.e.

$$
\Lambda_{\text {thickness }}=\frac{\partial}{\partial t} \ln d(\mathbf{x})
$$

Under the assumption of stochastic model (1), the thickness dilatation rate can be approximately distributed as Gausssian:

$$
\Lambda_{\text {thickness }}(\mathbf{x})=\lambda_{\text {thickness }}(\mathbf{x})+\epsilon_{\text {thickness }}(\mathbf{x})
$$

where $\lambda_{\text {thickness }}$ is the mean cortical thickness dilatation rate and $\epsilon_{\text {thickness }}$ is a mean zero Gaussian random field. Unlike the surface area change metric, cortical thickness can only be defined locally but we can compute the within average thickness dilatation rate for subject j:

$$
\Lambda_{\text {avg thickness }}^{j}=\frac{1}{\left\|\partial \Omega_{0}\right\|} \int_{\mathbf{x} \in \partial \Omega_{0}} \Lambda_{\text {thickness }}^{j}(\mathbf{x}) d \mathbf{x}
$$




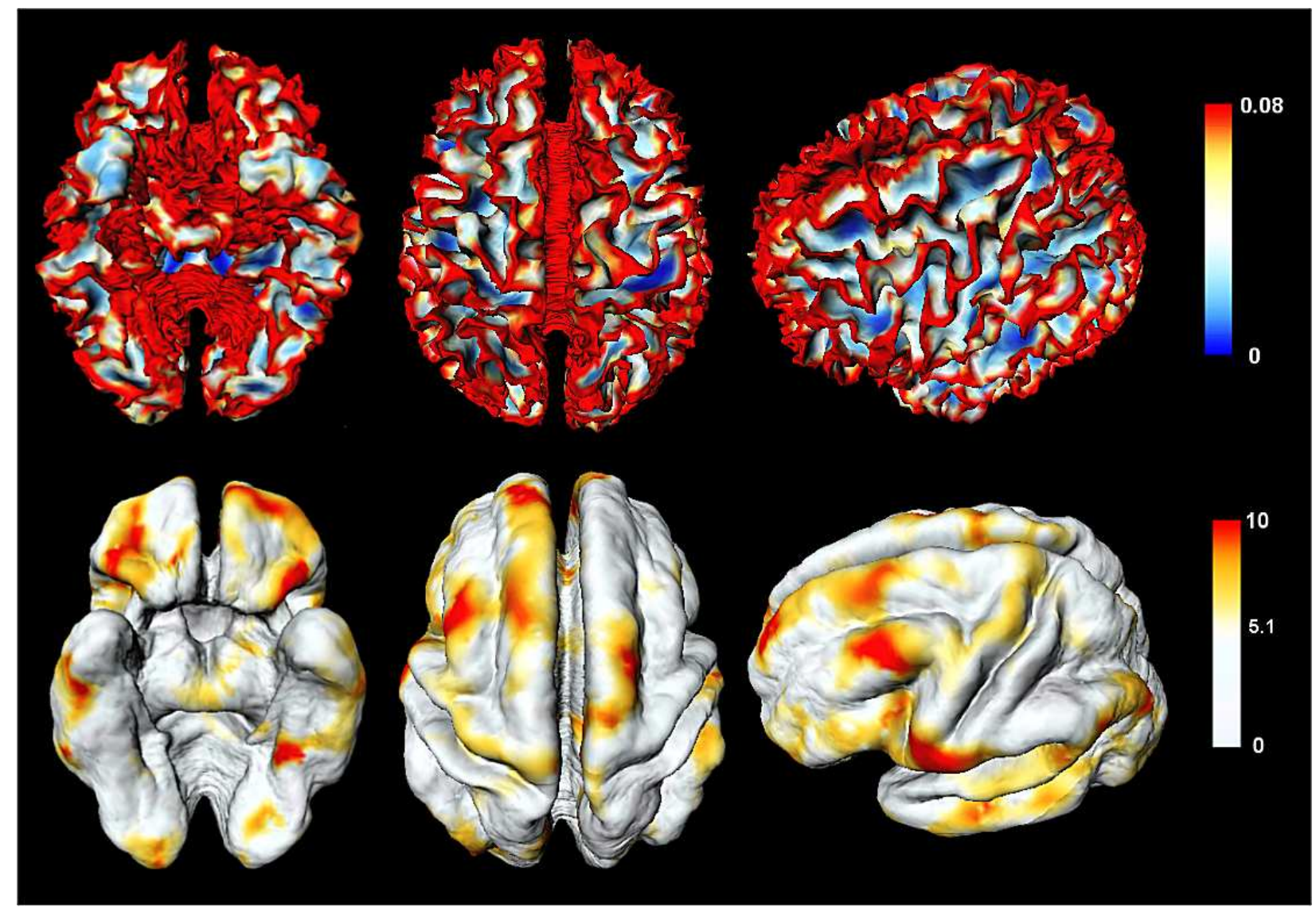

Figure 6: Top: Bending metric computed on the inner cortical surface of a 14 year old subject. It measures the amount of folding or curvature of the cortical surface. This metric can be also used to extract sulci and gyri in the problem of sulcal segmentation (see Figure 3). Bottom: Corrected $t$ map thresholded at 5.1 showing statistically significant region of curvature increase. Most of curvature increase occurs on gyri while there is no significant change of curvature on most of sulci. Also there is no statistically significant curvature decrease detected indicating that the complexity of the surface convolution may actually increase between ages 12 and 16 .

Then $\bar{\Lambda}_{\text {avg thickness }}$ will measure the between and within average cortical thickness dilatation rate.

\subsection{Curvature Change}

When the surface $\partial \Omega_{0}$ deforms to $\partial \Omega_{t}$, curvatures of the surface change as well. The principal curvatures can characterize the shape and location of the sulci and gyri, which are the valleys and crests of the cortical surfaces (Bartesaghi et al., 2001; Joshi et al., 1995; Khaneja et al., 1998; Subsol, 1999). By measuring the curvature changes, rapidly folding and cortical regions 
can be localized. Let $\kappa_{1}$ and $\kappa_{2}$ be the two principal curvatures as defined in Boothby (1986) and Kreyszig (1959). The principal curvatures can be represented as functions of $\beta_{i} \mathrm{~s}$ in quadratic surface (2) (Chung, 2001). To measure the amount of folding, we define bending metric $K$ as a function of the principal curvatures:

$$
K=\frac{\kappa_{1}^{2}+\kappa_{2}^{2}}{2}+\alpha
$$

We may arbitraly set $\alpha=0.001$. If the cortical surface is flat, bending metric $K$ obtains the minimum 0.001. The larger the bending metric, the more surface will be crested as shown in Figure 6.

We define the local curvature dilatation rate as

$$
\Lambda_{\text {curvature }}=\frac{\partial}{\partial t} \ln K
$$

Under the linear model (1), it can be similarly shown that the curvature dilatation is approximately distributed as a Gaussian random field (Chung et al., 2002):

$$
\Lambda_{\text {curvature }}(\mathbf{x})=\lambda_{\text {curvature }}(\mathbf{x})+\epsilon_{\text {curvature }}(\mathbf{x}),
$$

where $\lambda_{\text {curvature }}$ is the mean curvature dilatation rate and $\epsilon_{\text {curvature }}$ is a mean zero Gaussian random field. As in the case of the average cortical thickness dilatation rate (6), the average curvature dilatation rate can be computed and used as a global measure:

$$
\Lambda_{\text {avg curvature }}=\frac{1}{\left\|\partial \Omega_{0}\right\|} \int_{\partial \Omega_{0}} \Lambda_{\text {curvature }}(\mathbf{x}) d \mathbf{x} .
$$

A similar integral approach has been taken to measure the amount of bending in the 2D contour of the corpus callosum (Peterson et al., 2001).

\section{Surface-Based Diffusion Smoothing}

In order to increase the signal-to-noise ratio (SNR) as defined in Dougherty (1999), Rosenfeld and Kak (1982) and Worsley et al. (1996b), Gaussian kernel smoothing is desirable in many statistical analyses. For example, Figure 5 shows fairly large variations in cortical thickness of a single subject displayed on the average brain atlas $\Omega_{\text {atlas }}$. By smoothing the data 
on the cortical surface, the SNR will increase if the signal itself is smooth and in turn, it will be easier to localize the morphological changes. However, due to the convoluted nature of the cortex whose geometry is non-Euclidean, we can not directly apply Gaussian kernel smoothing on the cortical surface. Gaussian kernel smoothing of functional data $f(\mathbf{x}), \mathbf{x}=\left(x_{1}, \ldots, x_{n}\right) \in \mathbb{R}^{n}$ with FWHM (full width at half maximum) $=4(\ln 2)^{1 / 2} \sqrt{t}$ is defined as the convolution of the Gaussian kernel with $f$ :

$$
F(\mathbf{x}, t)=\frac{1}{(4 \pi t)^{n / 2}} \int_{\mathbb{R}^{n}} e^{-(x-y)^{2} / 4 t} f(y) d y
$$

Formulation (8) can not be directly to the cortical surfaces. However, by reformulating Gaussian kernel smoothing as a solution of a diffusion equation on a Riemannian manifold, the Gaussian kernel smoothing approach can be generalized to an arbitrary curved surface. This generalization is called diffusion smoothing and has been used in the analysis of fMRI data on the cortical surface (Andrade et al., 2001). It can be shown that (8) is the integral solution of the $n$-dimensional diffusion equation

$$
\frac{\partial F}{\partial t}=\Delta F
$$

with the initial condition $F(\mathbf{x}, 0)=f(\mathbf{x})$, where $\Delta=\partial^{2} / \partial x_{1}^{2}+\cdots+\partial^{2} / \partial x_{n}^{2}$ is the Laplacian in $n$-dimensional Euclidean space (Egorov and Shubin, 1991). Hence the Gaussian kernel smoothing is equivalent to the diffusion of the initial data $f(\mathbf{x})$ after time $t$. When applying diffusion smoothing on curved surfaces, the smoothing somehow has to incorporate the geometrical features of the curved surface and the Laplacian $\Delta$ should change accordingly. The extension of the Euclidean Laplacian to an arbitrary Riemannian manifold is called the Laplace-Beltrami operator (Arfken, 2000; Kreyszig, 1959). The approach taken in Andrade et al. (2001) is based on a local flattening of the cortical surface and estimating the planar Laplacian, which may not be as accurate as our estimation based on the finite element method (FEM). Further, our direct FEM approach completely avoid any local or global surface flattening. For given Riemannian metric tensor $g_{i j}$, the Laplace-Beltrami operator $\Delta$ is defined as

$$
\Delta F=\sum_{i, j} \frac{1}{|g|^{1 / 2}} \frac{\partial}{\partial u^{i}}\left(|g|^{1 / 2} g^{i j} \frac{\partial F}{\partial u^{j}}\right)
$$




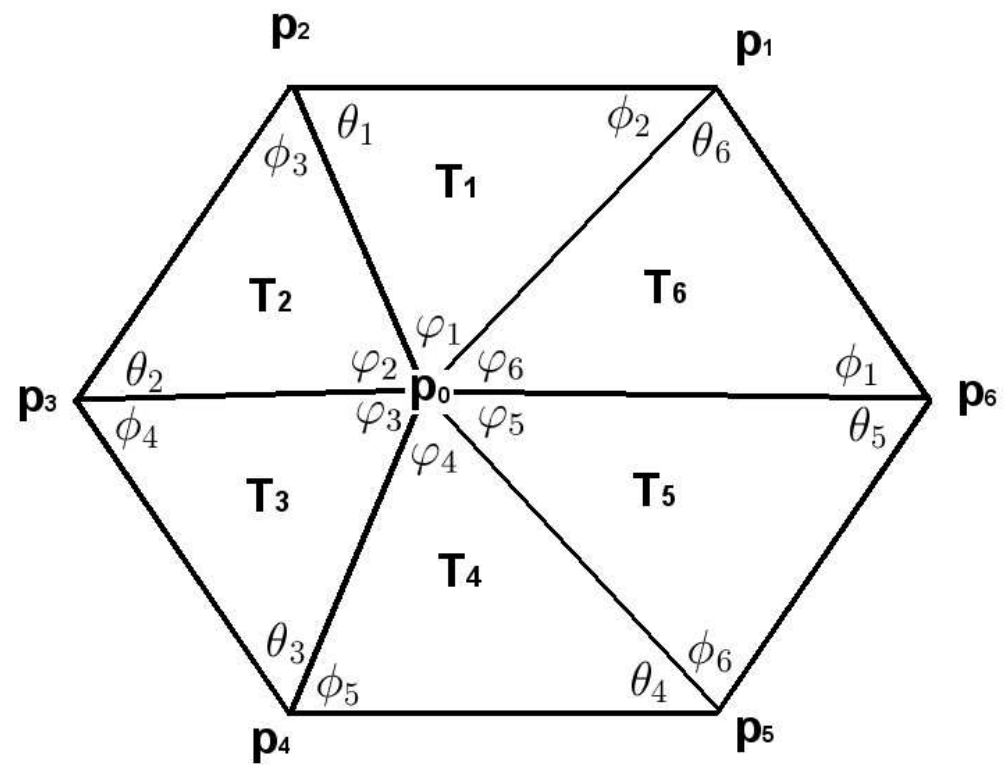

Figure 7: A typical triangulation in the neighborhood of $\mathbf{p}=\mathbf{p}_{0}$. When ASP algorithm is used, the triangular mesh is constructed in such a way that it is always pentagonal or hexagonal.

where $\left(g^{i j}\right)=g^{-1}$ (Arfken, 2000, pp. 158-167). Note that when $g$ becomes a $2 \times 2$ identity matrix, the Laplace-Beltrami operator in (10), simplifies to a standard 2D Laplacian:

$$
\Delta F=\frac{\partial^{2} F}{\partial\left(u^{1}\right)^{2}}+\frac{\partial^{2} F}{\partial\left(u^{2}\right)^{2}} .
$$

Using the FEM on the triangular cortical mesh generated by the ASP algorithm, it is possible to estimate the Laplace-Beltrami operator as the linear weights of neighboring vertices (Chung, 2001).

Let $\mathbf{p}_{1}, \cdots, \mathbf{p}_{m}$ be $m$ neighboring vertices around the central vertex $\mathbf{p}=\mathbf{p}_{0}$. Then the estimated Laplace-Beltrami operator is given by

$$
\widehat{\Delta F}(\mathbf{p})=\sum_{i=1}^{m} w_{i}\left(F\left(\mathbf{p}_{i}\right)-F(\mathbf{p})\right)
$$

with the weights

$$
w_{i}=\frac{\cot \theta_{i}+\cot \phi_{i}}{\sum_{i=1}^{m}\left\|T_{i}\right\|}
$$

where $\theta_{i}$ and $\phi_{i}$ are the two angles opposite to the edge connecting $\mathbf{p}_{i}$ and $\mathbf{p}$, and $\left\|T_{i}\right\|$ is the area of the $i$-th triangle (Figure 7). 

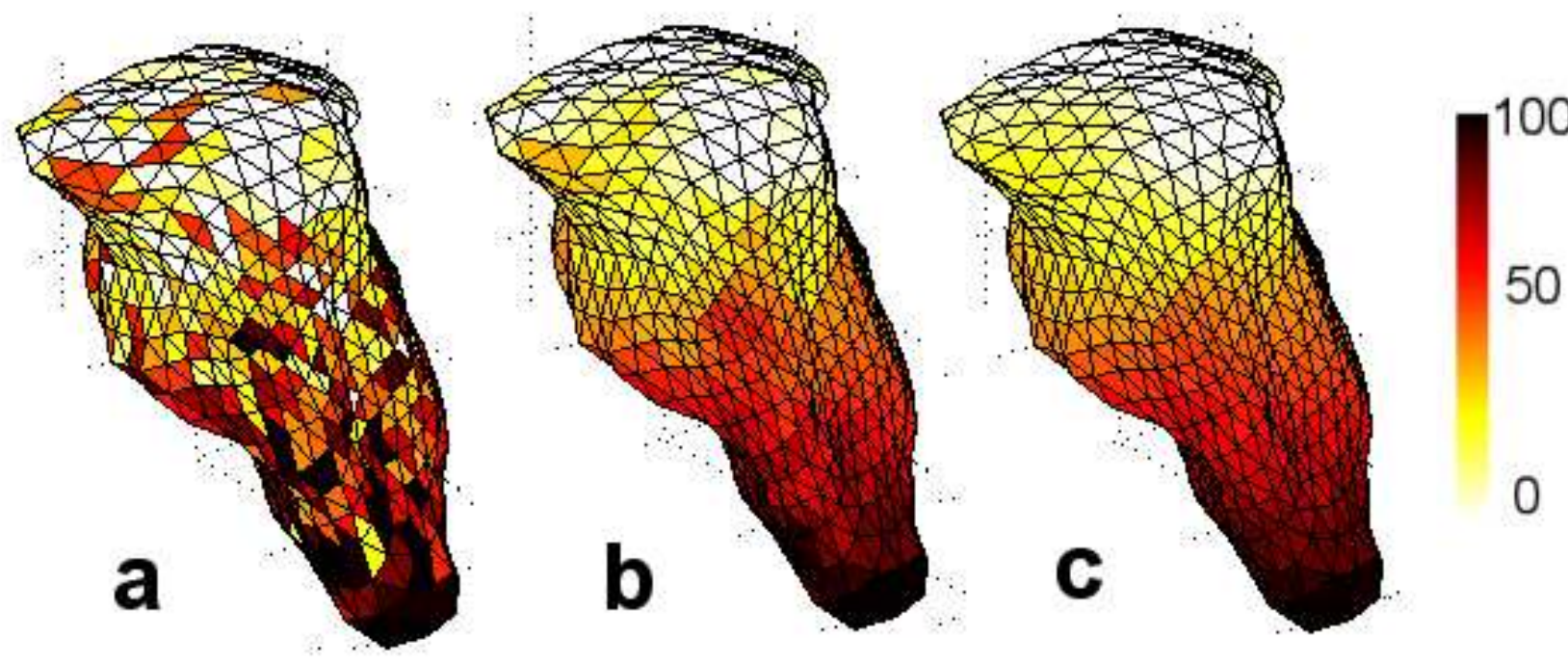

Figure 8: Diffusion smoothing simulation on a triangular mesh consisting of 1280 triangles. This smaller mesh is the surface of the brain stem. The artificial signal was generated with Gaussian noise to illustrate the smoothing process. (a) The initial signal. (b) After 10 iterations with $\delta t=0.5$. (c) After 20 iterations with $\delta t=0.5$.

This is an improved formulation from the previous attempt in diffusion smoothing on the cortical surface (Andrade et al., 2001), where the Laplacian is simply estimated as the planar Laplacian after locally flattening the triangular mesh consisting of nodes $\mathbf{p}_{0}, \cdots, \mathbf{p}_{m}$ onto a flat plane. In the numerical implementation, we have used formula

$$
\cot \theta_{i}=\frac{\left\langle\mathbf{p}_{i+1}-\mathbf{p}, \mathbf{p}_{i+1}-\mathbf{p}_{i}\right\rangle}{2\left\|T_{i}\right\|}, \cot \phi_{i}=\frac{\left\langle\mathbf{p}_{i-1}-\mathbf{p}, \mathbf{p}_{i-1}-\mathbf{p}_{i}\right\rangle}{2\left\|T_{i}\right\|}
$$

and $\left\|T_{i}\right\|=\left\|\left(\mathbf{p}_{i+1}-\mathbf{p}\right) \times\left(\mathbf{p}_{i}-\mathbf{p}\right)\right\| / 2$. Afterwards, the finite difference scheme is used to iteratively solve the diffusion equation at each vertex $\mathbf{p}$ :

$$
\frac{F\left(\mathbf{p}, t_{n+1}\right)-F\left(\mathbf{p}, t_{n}\right)}{t_{n+1}-t_{n}}=\widehat{\Delta} F\left(\mathbf{p}, t_{n}\right),
$$

with the initial condition $F(\mathbf{p}, 0)=f(\mathbf{p})$. After $N$-iterations, the finite difference scheme gives the diffusion of the initial data $f$ after duration $N \delta t$. If the diffusion were applied to Euclidean space, it would be equivalent to Gaussian kernel smoothing with

$$
\mathrm{FWHM}=4(\ln 2)^{1 / 2} \sqrt{N \delta t}
$$




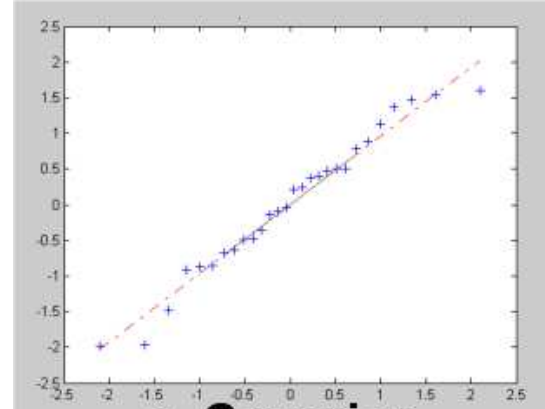

a. Gaussian

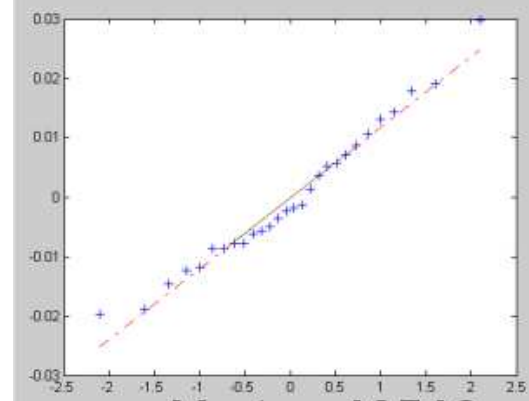

c. Vertex 40546

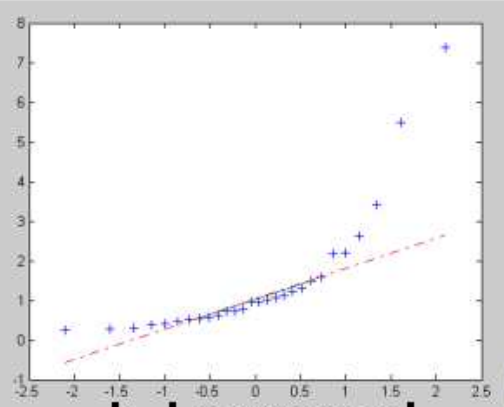

b. Lognormal

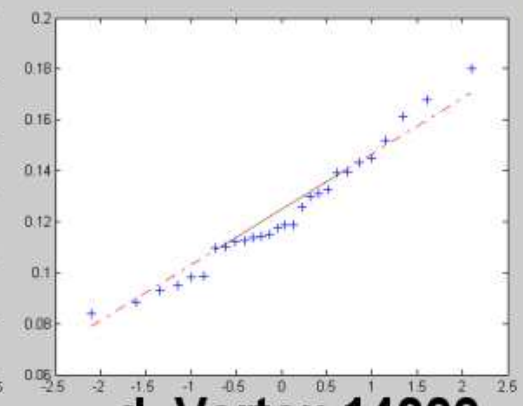

d. Vertex 14300

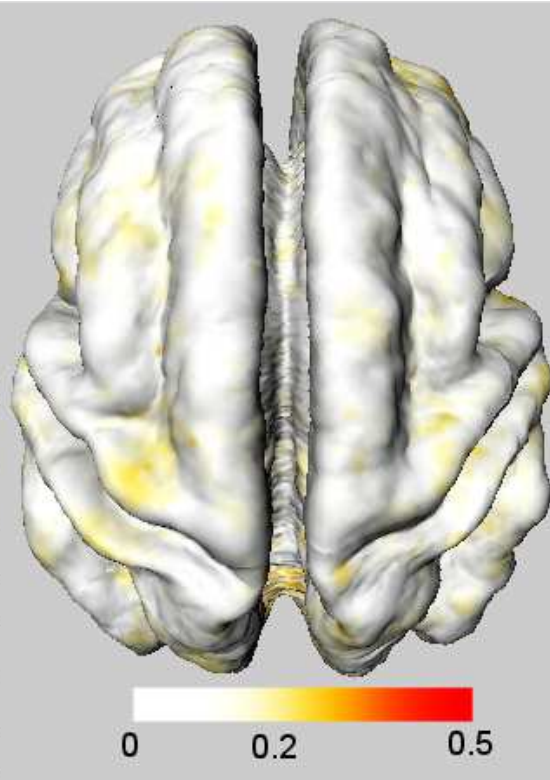

e. Lilliefors Statistic

Figure 9: Checking Gaussian assumption for the cortical thickness metric. The horizontal axis displays the qauntiles of a Gaussian distribution while the vertical axis displays the qauntiles of an empirical distribution. How closely the blue dots lie along the straight line gives an idea if the underlying empirical distribution follows the theoritical Gaussian distribution. (a) Computer simulation of a Gaussian distribution. (b) Computer simulation of a lognormal distribution. This is to illustrate how the qqplot of a non Gaussian distribution is different. (c) Vertex 40546 is where $t$ value is 10.2. (d) Vertex 14300 gives $t=27$. (e) Lilliefors statistic measures the maximum difference between the empirical and a theoretical Gaussian distributions. Most of cortex shows value less than the cutoff value 0.19 indicating that the Gaussian random field assumption is valid.

Computing the linear weights for the Laplace-Beltrami operator takes a fair amount of time (about 4 minutes in Matlab running on a Pentium III machine), but once the weights are computed, it is applied through the whole iteration repeatedly and the actual finite difference scheme takes only two minutes for 100 iterations. Figure 8 illustrates the process of diffusion smoothing. Unlike Gaussian kernel smoothing, smoothing is an iterative procedure. However, it should be emphasized that Gaussian kernel smoothing is a special case of diffusion smoothing restricted to Euclidean space. 


\section{Statistical Inference on the Cortical Surface}

All of our morphological measures such as surface area, cortical thickness, curvature dilatation rates are modeled as Gaussian random fields on the cortical surface, i.e.

$$
\Lambda(\mathbf{x})=\lambda(\mathbf{x})+\epsilon(\mathbf{x}), \mathbf{x} \in \partial \Omega_{\text {atlas }}
$$

where the deterministic part $\lambda$ is the mean of the metric $\Lambda$ and $\epsilon$ is a mean zero Gaussian random field. This theoretical model assumption has been checked using both Lilliefors test (Conover, 1980) and quantile-quantile plots (qqplots) (Hamilton, 1992). The qqplot displays quantiles from an empirical distribution on the vertical axis versus theoretical quantiles from a Gaussisan distribution on the horizontal axis. It is used to check graphically if the empirical distribution follows the theoretical Gaussian distribution. If the data comes from a Gaussian field, then the qqplot should be close to a straight line (Figure 9 (a)). If the data comes from a lognormal distribution, it may not form a straight line (Figure 9 (b)). Because it is not possible to view qqplots for every vertices on the cortex, we measured the correlation coefficients $\gamma$ of the vertical and horizontal coordinates in qqplots. If the empirical distribution comes from Gaussian, $\gamma$ should asymptotically converge to 1. For Gaussian simulation, $\gamma=0.98 \pm 0.01$ and for lognormal simulation $\gamma=0.84 \pm 0.08$ on the cortex. For the cortical thickness data, which has been filtered with the diffusion smoothing, $\gamma=0.96 \pm 0.03$. So it does seems that the smoothed cortical thickness metric can be modeled as a Gaussian random field. Using Lilliefors statistic, we statistically tested the Gaussian assumption. The Lilliefors test, which is a special case of the Komogorov-Smirnov test, looks at the maximum difference between the empirical and a theoretical Gaussian distribution when the mean and the variance of the distribution are not known. Since the Lilliefors statistics of the cortical thickness metric are mostly smaller than the cutoff value of 0.19 at $1 \%$ level $(0.16$ at $5 \%$ level), there is no reason to reject model (11). (Figure $9(\mathrm{e})$ ).

Gaussian kernel smoothed images tend to reasonably follow random field assumptions when a fairly large FWHM is used. Diffusion smoothing is equivalent to Gaussian kernel smoothing locally in conformal coordinates on the cortex (Chung, 2001b). Also P-value for local maxima formula is quite stable even if a slightly different assumption such as nonisotropy is used (Worsley et al., 1999b). Therefore, detecting the region of statistically 
significant $\lambda(\mathbf{x})$ for some $\mathbf{x}$ can be done via thresholding the maximum of the $T$ random field defined on the cortical surface (Worsley et al., 1996a; Worsley et al., 1999).

The $T$ random field on the manifold $\partial \Omega_{\text {atlas }}$ is defined as

$$
T(\mathbf{x})=\sqrt{n} \frac{M(\mathbf{x})}{S(\mathbf{x})}, \mathbf{x} \in \partial \Omega_{\text {atlas }}
$$

where $M$ and $S$ are the sample mean and standard deviation of metric $\Lambda$ over the $n$ subjects. Under the null hypothesis

$$
H_{0}: \lambda(\mathbf{x})=0 \text { for all } \mathbf{x} \in \partial \Omega_{\text {atlas }}
$$

i.e. no structural change, $T(\mathbf{x})$ is distributed as a student's $t$ with $n-1$ degrees of freedom at each voxel $\mathbf{x}$. The $P$ value of the local maxima of the $T$ field will give a conservative threshold, which has been used in brain imaging for a quite some time (Worsley, 1996a).

For very high threshold $y$, we can show that

$$
P\left(\max _{\mathbf{x} \in \partial \Omega_{\text {atlas }}} T(\mathbf{x}) \geq y\right) \approx \sum_{i=0}^{3} \phi_{i}\left(\partial \Omega_{\text {atlas }}\right) \rho_{i}(y),
$$

where $\rho_{i}$ is the $i$-dimensional EC-density and the Minkowski functional $\phi_{i}$ are

$$
\phi_{0}\left(\partial \Omega_{\text {atlas }}\right)=2, \phi_{1}\left(\partial \Omega_{\text {atlas }}\right)=0, \phi_{2}\left(\partial \Omega_{\text {atlas }}\right)=\left\|\partial \Omega_{\text {atlas }}\right\|, \phi_{3}\left(\partial \Omega_{\text {atlas }}\right)=0
$$

and $\left\|\partial \Omega_{\text {atlas }}\right\|$ is the total surface area of $\partial \Omega_{\text {atlas }}$ (Worsley, 1996a). When diffusion smoothing with given FWHM is applied to metric $\Lambda$ on surface $\partial \Omega_{\text {atlas }}$, the 0 -dimensional and 2-dimensional EC-density becomes

$$
\begin{gathered}
\rho_{0}(y)=\int_{y}^{\infty} \frac{\Gamma\left(\frac{n}{2}\right)}{((n-1) \pi)^{1 / 2} \Gamma\left(\frac{n-1}{2}\right)}\left(1+\frac{y^{2}}{n-1}\right)^{-n / 2} d y, \\
\rho_{2}(y)=\frac{1}{\mathrm{FWHM}^{2}} \frac{4 \ln 2}{(2 \pi)^{3 / 2}} \frac{\Gamma\left(\frac{n}{2}\right)}{\left(\frac{n-1}{2}\right)^{1 / 2} \Gamma\left(\frac{n-1}{2}\right)} y\left(1+\frac{y^{2}}{n-1}\right)^{-(n-2) / 2} .
\end{gathered}
$$

Therefore, the excursion probability on the cortical surface can be approximated by the following formula:

$$
P\left(\max _{\mathbf{x} \in \partial \Omega_{\text {atlas }}} T(\mathbf{x}) \geq y\right) \approx 2 \rho_{0}(y)+\left\|\partial \Omega_{\text {atlas }}\right\| \rho_{2}(y)
$$

We compute the total surface area $\left\|\partial \Omega_{\text {atlas }}\right\|$ by summing the area of each triangle in a triangulated surface. The total surface area of the average atlas brain is $275,800 \mathrm{~mm}^{2}$, which 


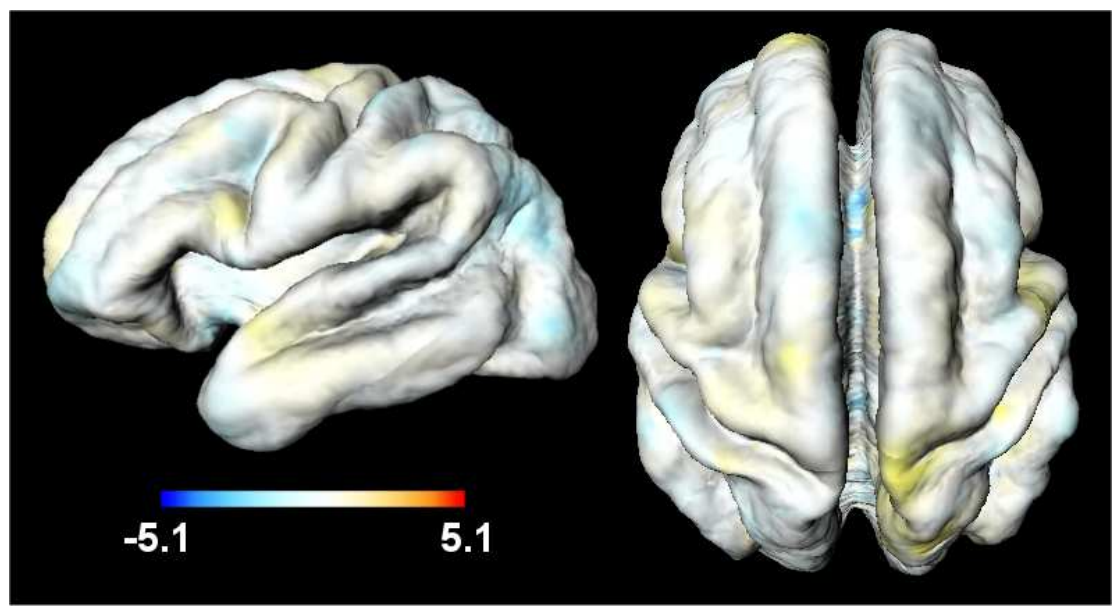

Figure 10: T-map of the cortical dilatation rate for null data. The null data was created by reversing time for the half of subjects chosen randomly. In the null data, the mean time difference is -0.24 year, so our statistical analysis should not detect any morphological changes. In fact, the $t$-values in every vertices were well below the threshold.

is roughly the area of $53 \times 53 \mathrm{~cm}^{2}$ sheet. We want to point out that the surface area of the average atlas brain is not the average surface area of 28 subjects. When $20 \mathrm{~mm}$ FWHM diffusion smoothing is used on the template surface $\partial \Omega_{\text {atlas }}, 2.5 \%$ thresholding gives

$$
P\left(\max _{\mathbf{x} \in \partial \Omega_{\text {atlas }}} T(\mathbf{x}) \geq 5.1\right)=P\left(\max _{\mathbf{x} \in \partial \Omega_{\text {atlas }}} T(\mathbf{x}) \leq-5.1\right) \approx 0.025
$$

Our surface-based smoothing and analysis are checked on null data. The null data is created by reversing time for randomly chosen half of the subjects. In the null data, the mean time difference $t_{2}-t_{1}$ is -0.24 year so the statistical analysis presented here should not detect any morphological changes. For the cortical thickness dilatation rate, the maximum and the minimum t-values are 3.1808 and -3.9570 respectively, well bellow the threshold 5.1 and -5.1 indicating that the analysis obviously did not detect any statistically significant morphological changes.

\section{Results}

Twenty-eight normal subjects were selected based on the same physical, neurological and psychological criteria described in Giedd et al. (1996). This is the same data set reported in Chung et al. (2001), where the Jacobian of the 3D deformation was used to detect 
statistically significant brain tissue growth or loss in 3D whole brain via deformation-based morphometry. 3D Gaussian kernel smoothing used in this study is not sensitive to the interfaces between the gray, white matter and CSF. Gaussian kernel smoothing tends to blur gray matter volume increase data across the cortical boundaries. So in some cases, statistically significant brain tissue growth could be found in CSF. Our deformation-based surface morphometry can overcome this inherent shortcoming associated with the previous morphometric analysis.

Two $\mathrm{T}_{1}$-weighted MR scans were acquired for each subject at different times on the same GE Sigma 1.5-T superconducting magnet system. The first scan was obtained at the age $t_{1}=11.5 \pm 3.1$ years (min 7.0 years, max 17.8 years) and the second scan was obtained at the age $t_{2}=16.1 \pm 3.2$ years ( $\min 10.6$ years, $\max 21.8$ years). The time difference between the first and the second scan was $4.6 \pm 0.9$ years (min time difference 2.2 years , max time difference 6.4 years). Using the automatic image processing pipeline (Zijdenbos et al., 1998), MR images were spatially normalized into standardized stereotactic space via a global affine transformation (Collins et al., 1994; Talairach and Tournoux, 1988). Subsequently, an automatic tissue-segmentation algorithm based on a supervised artificial neural network classifier was used to classify each voxel as CSF, gray matter and white matter (Vasken, 1996). Afterwards, a triangular mesh for each cortical surface was generated by deforming a mesh to fit the proper boundary in a segmented volume using the ASP algorithm. As described in the previous section, the ASP algorithm is used to extract the surface and compute the displacement field on the outer cortical surface. Then we computed the local area dilatation, the cortical thickness and the curvature dilatation rates. Such surface metrics are then filtered with $20 \mathrm{~mm}$ FWHM diffusion smoothing. Image smoothing should improves the power of detection and compensates for some of registration errors (Chung et al., 2001).

Gray matter volume change: The total gray matter volume dilatation rate for each subject was computed by computing the volume of triangular prisms that forms gray matter. The mean total gray matter volume dilatation rate $\bar{\Lambda}_{\text {total volume }}=-0.0050$. This $0.5 \%$ annual decrease in the total gray matter volume is statistically significant ( $t$ value of -4.45$)$. There has been substantial developmental studies on gray matter volume reduction for children and adolescents (Courchesne et al, 2000; Giedd et al., 1999; Jernigan et al., 1991; Pfefferbaum et 


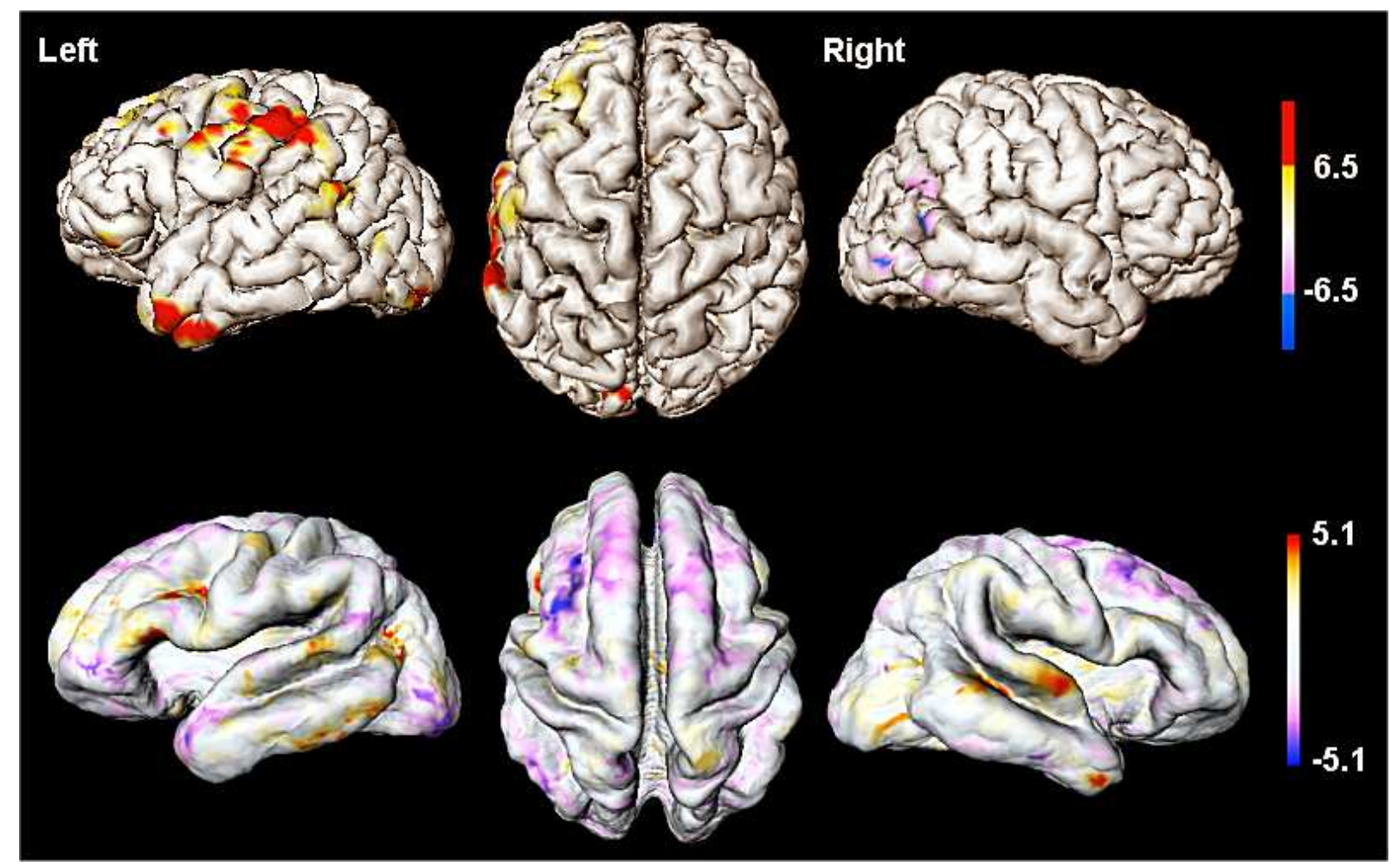

Figure 11: Top: $t$ map of local gray matter change computed in $3 \mathrm{D}$ and then superimposed with an atlas brain. The predominant local gray matter volume increases were detected in somatosensory and motor cortex and temporal lobe. The left and right hemispheres show asymmetric growth pattern. Bottom: $t$ map of the cortical surface area dilatation rate showing the statistically significant region of area expansion and reduction. The red regions are statistically significant surface area expansions while the blue regions are statistically significant surface area reductions between ages 12 and 16.

al., 1994; Rajapakse et al., 1996; Riess et al., 1996; Steen et al., 1997). Our result confirms these studies. However, the ROI-based volumetry used in the previous studies did not allow investigators to detect local volume change within the ROIs. Our local volumetry based on deformation field can overcome the limitation of the ROI-based volumetry.

Brain tissue growth and loss based on the local volume dilatation rate was detected in the whole brain volume that includes both gray and white matter (Chung et al., 2001). The morphometric analysis used in Chung et al. (2001) generates 3D statistical parametric map (SPM) of brain tissue growth. By superposing the 3D SPM with the triangular mesh of the cortical surface of the atlas brain, we get gray matter volume change SPM restricted onto the cortical surface (Figure 11). Although it is an ad hoc approach, the resulting SPM projected on to the atlas brain seem to confirm some of the results in Giedd et al. (1999) 
and Thompson et al. (2000). In particular, Giedd et al. (1999) reported that frontal and parietal gray matters decrease but temporal and occipital gray matters increase even after age 12. In our analysis, we found local gray matter volume growth in the parts of temporal, occipital, somatosensory and motor regions but did not detect any volume loss in the frontal lobe. Instead we found statistically significant structural movements without accompanying volume decreases (Chung et al. 2001).

Surface area change: We measured the total surface area dilatation rate for each subject by computing the total area of triangular meshes on the both outer and inner cortical surfaces. The mean surface areas for all 28 subjects area are

$$
\begin{gathered}
\left\|\partial \Omega_{t_{1}}^{\text {out }}\right\|=302180 \mathrm{~mm}^{2},\left\|\partial \Omega_{t_{1}}^{\text {in }}\right\|=289380 \mathrm{~mm}^{2} \\
\left\|\partial \Omega_{t_{2}}^{\text {out }}\right\|=229940 \mathrm{~mm}^{2},\left\|\partial \Omega_{t_{2}}^{\text {in }}\right\|=221520 \mathrm{~mm}^{2}
\end{gathered}
$$

So we can see that both the outer and inner surface area tend to decrease between ages 12 and 16 . The mean total area dilatation rate for 28 subjects was found to be

$$
\bar{\Lambda}_{\text {total area }}^{\text {out }}=-0.0093, \bar{\Lambda}_{\text {total area }}^{\text {in }}=-0.0081 .
$$

0.8 to $0.9 \%$ decrease of both surface area change per year is statistically significant $(t$ value of -9.2 and -7.5 respectively). So it does seem that the outer surface shrinks faster than the inner surface.

After knowing that the total surface areas shrink, we need to identify the regions of local surface area growth or reduction. The surface area dilatation rates were computed for all subjects, then smoothed with 20mm FWHM diffusion smoothing to increase the signal-tonoise ratio. Averaging over 28 subjects, local surface area change was found to be between $-15.79 \%$ and $13.78 \%$ per year. In one particular subject, we observed between $-106.5 \%$ and $120.3 \%$ of the local surface area change over 4 year time span. Figure 11 is the $t$ map of the cortical surface area dilatation showing cortical tissue growth pattern. Surface area growth and decrease were detected by $T>5.1$ and $T<-5.1(P<0.05$, corrected $)$ respectively, showing statistically significant local surface expansion in Broca's area in the left hemisphere and local surface shrinkage in the left superior frontal sulcus. Most of surface reduction seems to be concentrated near the frontal region. 
Cortical thickness change: The growth pattern of cortical thickness change is found to be very different but closely related to that of local surface area change. The average cortical thickness at $t_{1}$ is $2.55 \mathrm{~mm}$ while the average cortical thickness at $t_{2}$ is $2.60 \mathrm{~mm}$. The average cortical thickness dilatation rate across all within and between subjects was found to be $\bar{\Lambda}_{\text {avg thickness }}=0.012$. This $1.2 \%$ annual increase in the cortical thickness is statistically significant ( $t$ value of 4.86). However, three out of 28 subjects showed from 0.6 up to $2 \%$ of cortical thinning. Although there are regions of cortical thinning present in all individual subjects, our statistical analysis indicates that the overall global pattern of thickness increase is more predominant feature between ages 12 and 16. Also we localized the region of statistically significant cortical thickness increase by thresholding the $t$ map of the cortical thickness dilatation rate by 5.1 (Figure 5). Cortical thickening is widespread on the cortex. The most predominant thickness increase was detected in the left superior frontal sulcus, which is the same location we detected local surface area reduction while local gray matter volume remains the same. So it seems that while there is no gray matter volume change, the left superior frontal sulcus undergoes cortical thickening and surface area shrinking and perhaps this is why we did not detect any local volume change in this region.

The most interesting result found so far is that there is almost no statistically significant local cortical thinning detected on the whole cortical surface between ages 12 and 16 . As we have shown, the total inner and outer surface areas as well as the total volume of gray matter decrease. So it seems that all these results are in contradiction. However, if the rate of the total surface area decrease is faster than the rate of the total volume reduction, it is possible to have cortical thickening. To see this, suppose we have a shallow solid shell with constant thickness $h$, total volume $V$ and total surface area $A$. Then $V=h A$. It can be shown that the rate of volume change per unit volume can be written as $\dot{V} / V=$ $\dot{h} / h+\dot{A} / A$. Using our dilatation notation, $\bar{\Lambda}_{\text {total volume }}=\bar{\Lambda}_{\text {avg thickness }}+\bar{\Lambda}_{\text {total area }}^{\text {out }}$. In our data, $\bar{\Lambda}_{\text {total volume }}=-0.0050>\bar{\Lambda}_{\text {total area }}^{\text {out }}=-0.0093$, so we should have increase in the cortical thickness. However, we want to point out that this argument is heuristic because the cortical thickness is not uniform across the cortex. Sowell et al. (2001) reported cortical thinning or gray matter density decrease in the frontal and parietal lobes in similar age group. The thickness measure they used is based on gray matter density, which measures 
the proportion of gray matter within a sphere with fixed radius between 5 to $15 \mathrm{~mm}$ around a point on the outer cortical surface (Sowell et al., 2001; Thompson et al., 2001). However, the gray matter density not only measures the cortical thickness but also the amount of bending. If a point is chosen on a gyrus, the increase in the bending energy will correspond to the increase in gray matter density. So the region of gray matter density decrease reported in Sowell et al. (2001) more closely resembles the region of curvature increase (Figure 6) than the region of cortical thickness change (Figure 5). Because they measure different anatomical quantities, it is hard to directly compare the result reported in Sowell et al. (2001) to our result.

Curvature change: Our study might be the first to use the curvature as the direct measure of anatomical changes in normal brain development. We measured curvature dilatation rate for each subject. The average curvature dilatation rate was found to be $\bar{\Lambda}_{\text {avg curvature }}=2.50 .250 \%$ increase is statistically significant ( $t$ value of 19.42). Local curvature change was detected by thresholding the $t$ statistic of the curvature dilatation rate at 5.1 (corrected). The superior frontal and middle frontal gyri show curvature increase. It is interesting to note that between these two gyri we have detected cortical thickness increase and local surface area decrease. It might be possible that cortical thickness increase and local surface area shrinking in the superior frontal sulcus causes the bending in the neighboring middle and superior frontal gyri. Such interacting dynamic pattern has been also detected in Chung et al. (2001), where gray matter tissue growth causes the inner surface to translate toward the region of white matter tissue reduction.

We also found no statistically significant local curvature decrease over the whole cortex. While the gray matter is shrinking in both total surface area and volume, the cortex itself seems to get folded to give increasing curvature.

\section{Conclusions}

The surface-based morphometry presented here can statistically localize the regions of cortical thickness, area and curvature change at a local level without specifying the regions of interest (ROI). This ROI-free approach might be best suitable for exploratory whole brain 
morphometric studies. Our analysis successfully avoids artificial surface flattening (Andrade et al., 2001; Angenent et al., 1999), which can destroy the inherent geometrical structure of the cortical surface. It seems that any structural or functional analysis associated with the cortex can be performed without surface flattening if an appropriate mathematics is used.

Our metric tensor formulation gives us an added advantage that not only it can be used to measure local surface area and curvature change of the cortex but also it is used for generalizing Gaussian kernel smoothing on the cortex via diffusion smoothing. Since it is a direct generalization of Gaussian kernel smoothing, the diffusion smoothing should locally inherit many mathematical and statistical properties of Gaussian kernel smoothing applied to standard 3D whole brain volume. The modification for any other triangular mesh can be easily done. We tried to combine and unify morphometric measurement, image smoothing and statistical inference in the same mathematical and statistical framework.

As an illustration of this powerful unified approach, we applied it to a group of normal children and adolescents to see if we can detect the region of anatomical changes in gray matter. It is found that the cortical surface area and gray matter volume shrink, while the cortical thickness and curvature tend to increase between ages 12 and 16 with a highly localized area of cortical thickening and surface area shrinking found in the superior frontal sulcus at the same time. It seems that the increase in thickness and the decrease in the superior frontal sulcus might cause increased folding in the middle and superior frontal gyri.

Our unified deformation-based surface morphometry can be also used as a tool for future investigations of neurodevelopmental disorders where surface analysis of either the cortex or brain substructures would be relevant.

\section{References}

[1] Andrade, A., Kherif, F., Mangin, J., Worsley, K.J., Paradis, A., Simon, O., Dehaene, S., Le Bihan, D., and Poline, J-B. (2001). Detection of Fmri Activation Using Cortical Surface Mapping. Human Brain Mapping, 12:79-93.

[2] Angenent, S., Hacker, S., Tannenbaum, A., and Kikinis, R. (1999). On the LaplaceBeltrami Operator and Brain Surface Flattening. IEEE Transactions on Medical Imag- 
ing, 18:700-711.

[3] Arfken, G.B. (2000). Mathematical Methods for Physicists. Academic Press, 5th. edition.

[4] Bartesaghi, A. and Sapiro, G. (2001). A System for the Generation of Vurves on 3D Brain Images. Human Brain Mapping, 14:1-15.

[5] Boothby, W.M. (1986). An Introduction to Differential Manifolds and Riemannian Geometry. Academic Press, London, 2nd. edition.

[6] Do Carmo, M.P. (1992). Riemannian Geometry. Prentice-Hall, Inc.

[7] Chung, M.K., Worsley, K.J., Paus, T., Cherif, D.L., Collins, C., Giedd, J., Rapoport, J.L., and Evans, A.C. (2001a). A Unified Statistical Approach to Deformation-Based Morphometry. NeuroImage, 14:595-606.

[8] Chung, M.K. (2001b). Statistical Morphometry in Computational Neuroanatomy. Ph.D.thesis, McGill University, Montreal, Canada. http://www.math.mcgill.ca/chung/theis/thesis.pdf.

[9] Chung, M.K., Worsley, K.J., Paus, T., Robbins, S., Taylor, J., Giedd, J., Rapoport, J.L., and Evans, A.C. (2002). Tensor-Based Surface Morphometry, Technical Report 1049, Department of Statistics, University of Wisconsin-Madison. http://www.stat.wisc.edu/ mchung/publications.html.

[10] Collins, D.L., Neelin, P., Peters, T.M., and Evans, A.C. (1994). Automatic 3D Intersubject Registration of MR Volumetric Data in Standardized Talairach Space. Journal of Computer Assisted Tomography, 18:192-205.

[11] Conover, W.J. (1980). Practical Nonparametric Statistics. New York, Wiley.

[12] Courchesne, E., Chisum, H.J., Townsend, J., Cowles, A., Covington, J., Egaas, B., Harwood, M., Hinds, S., and Press, G.A. (2000). Normal Brain Development and Aging: Quantitative Analysis at in Vivo MR Imaging in Healthy Volunteers. Radiology, 216:672-682. 
[13] Dale, A.M and Fischl, B. (1999). Cortical Surface-based Analysis I. Segmentation and Surface Reconstruction. NeuroImage, 9:179-194.

[14] Davatzikos, C. and Bryan, R.N. (1995). Using a Deformable Surface Model to Obtain a Shape Representation of the Cortex. In Proceedings of the IEEE International Conference on Computer Vision, pages 2122-2127.

[15] Dougherty, E.R. (1999). Random Processes for Image and Signal Processing. IEEE Press.

[16] Drew, D.A. (1991). Theory of Multicomponent Fluids. Springer-Verlag, New York.

[17] Egorov, Y.V. and Shubin, M.A. (1991). Partial Differential Equations I: Foundations of the Classical Theory. Springer-Verlag.

[18] Evans, A.C., Collins, D.L., Mills, S.R., Brown, E.D., Kelly, R.L., and Peters, T.M. (1993). 3D Statistical Neuroanatomical Models from 305 MRI Volumes. Proc. IEEENuclear Science Symposium and Medical Imaging Conference, 1813-1817.

[19] Giedd, J.N., Blumenthal, J., Jeffries, N.O., Rajapakse, J.C., Vaituzis, A.C., Liu, H., Berry, Y.C., Tobin, M., Nelson, J., and Castellanos, F.X. (1999). Development of the Human Corpus Callosum During Childhood and Adolescence: a Longitudinal MRI study. Progress in Neuro-Psychopharmacology \& Biological Psychiatry, 23:571-588.

[20] Giedd, J.N., Snell, J.W., Lange, N., Rajapakse, J.C., Kaysen, D., Vaituzis, A.C., Vauss, Y.C., Hamburger, S.D., Kozuch, P.L., and Rapoport, J.L. (1996). Quantitative Magnetic Resonance Imaging of Human Brain Development: Ages 4-18. Cerebral Cortex, 6:551-160.

[21] Good, C.D., Johnsrude, I.S., Ashburner, J., Henson, R.N., Friston, K.J., and Frackowiak, R.S.J. (2001). A Voxel-based Morphometric Study of Aging in 465 Normal Adult Human Brains. NeuroImage, 14:21-36.

[22] Griffin, L.D. (1994). The Intrinsic Geometry of the Cerebral Cortex. Journal of Theoretical Biology, 166:261-273. 
[23] Gurtin, M.E. and McFadden, G.B. (1994). On the Evolution of Phase Boundaries. Springer-Verlag, New York.

[24] Hamilton, L.C. (1992). Regression with Graphics. Wadsworth, Inc., Belmont, California.

[25] Henery, C. and Mayhew, T. (1989). The Cerebrum and Cerebellum of the Fixed Human Brain: Efficient and Unbiased Estimates of Volumes and Cortical Surface Areas. J. Anat., 167:167-180.

[26] Jernigan, T.L., Trauner, D.A., Hesselink, J.R., and Tallal, P.A. (1991). Maturation of Human Cerebrum Observed in Vivo During Adolescence. Brain, 114:2037-2049.

[27] Jones, S.E., Buchbinder, B.R., and Aharon, I. (2000). Three-dimensional Mapping of Cortical Thickness Using Laplace's Equation. Human Brain Mapping, 11:12-32.

[28] Joshi, S.C., Wang, J., Miller, M.I., Van Essen, D.C., and Grenander, U. (1995). On the Differential Geometry of the Cortical Surface. Vision Geometry IV, Vol. 2573, Proceedings of the SPIE's 1995 International Symposium on Optical Science, Engineering and Instrumentation, pages 304-311.

[29] Kabani, N., Le Goualher, G., MacDonald, D., and Evans, A.C. (2000). Measurement of Cortical Thickness Using an Automated 3-D Algorithm: A Validation Study. NeuroImage, 13:375-380.

[30] Khaneja, N., Miller, M.I., and Grenander, U. (1998). Dynamic Programming Generation of Curves on Brain Surfaces. IEEE Transactions on Pattern Analysis and Machine Intelligence, 20:1260-1265.

[31] Kreyszig, E. (1959). Differential Geometry. University of Toronto Press, Toronto, 1959.

[32] Lorensen, W.E. and Cline, H.E. (1987) Marching Cubes: A Hight Resolution 3D Surface Construction Algorithm. Computer Graphics, 21:21.

[33] MacDonald, J.D. (1997). A Method for Identifying Geometrically Simple Surfaces from Three Dimensional Images Ph. D. thesis, School of Computer Science, McGill University, Canada. 
[34] MacDonald, J.D., Kabani, N., Avis, D., and Evans, A.C. (2000). Automated 3-D Extraction of Inner and Outer Surfaces of Cerebral Cortex from MRI. NeuroImage, 12:340-356.

[35] Ozkan, M., Dawant, B.M., and Maciunas, R.J. (1993). Neural-Network-Based Segmentation of Multi-Modal Medical Images: a Comparative and Prospective Study. IEEE Trans. Med. Imaging, 12:534-544.

[36] Paus, T., Zijdenbos, A., Worsley, K.J., Collins, D.L., Blumenthal, J., Giedd, J.N., Rapoport, J.L., and Evans, A.C. (1999). Structural Maturation of Neural Pathways in Children and Adolescents: In Vivo Study. Science, 283:1908-1911.

[37] Peterson, B.S., Feineigle, F.A., Staib, L.H., and Gore, J.C. (2001). Automated Measurement of Latent Morphological Features in the Human Corpus Callosum. Human Brain Mapping, 12:232-245.

[38] Pfefferbaum, A., Mathalon, D.H., Sullivan, E.V., Rawles, J.M., Zipursky, R.B., and Lim, K.O. (1994). A Quantitative Magnetic Resonance Imaging Study of Changes in Brain Morphology from Infancy to Late Adulthood. Archives of Neurology, 51:874-887.

[39] Rajapakse, J.C., Giedd, J.N., DeCarli, C., Snell, J.W., McLaughlin, A., Vauss, Y.C., Krain, A.L., Hamburger, S., and Rapoport, J.L. (1996). A Technique for Single-Channel MR Brain Tissue Segmentation: Application to a Pediatric Sample. Magnetic Resonance Imaging, 14:1053-1065.

[40] Riess, A.L., Abrams, M.T., Singer, H.S., Ross, J.L., and Denckla, M.B. (1996). Brain Development, Gender and IQ in Children: A Volumetric Imaging Study. Brain, 119:1763-1774.

[41] Rosenfeld, A., and Kak., A. C. (1982) Digital Picture Processing, Vol. 2. Academic Press, Orlando.

[42] Sethian., J. A. (1996). Level Set Methods and Fast Marching Methods. Cambridge University Press. 
[43] Sled, J.G., Zijdenbos, A.P., and Evans, A.C. (1998). A Nonparametric Method for Automatic Correction of Intensity Nonuniformity in MRI Data. IEEE Transactions on Medical Imaging, 17:87-97.

[44] Sowell, E.R., Thompson, P.M., Tessner, K.D., and Toga, A.W. (2001). Mapping Continued Brain Growth and Gray Matter Density Reduction in Dorsal Frontal Cortex: Inverse Relationships During Postadolescent Brain Maturation. The Journal of Neuroscience, 21:8819-8829.

[45] Steeen, R.G., Ogg, R.J., Reddick, W.E., and Kingsley, P.B. (1997). Age-related Changes in the Pediatric Brain: Quantitative MR Evidence of Maturational Changes during Adolescence. Am. J. Neuroradiol., 18:819-828.

[46] Subsol, G. (1999). Crest Lines for Curve-Based Warping. Brain Warping, pages 241262.

[47] Talairach, J., and Tournoux., P. (1988). Co-planar Stereotactic Atlas of the Human Brain: 3-Dimensional Proportional System. an Approach to Cerebral Imaging. Thieme, Stuttgart.

[48] Thompson, P.M., Giedd, J.N., Woods, R.P., MacDonald, D., Evans, A.C., and Toga, A.W. (2000). Growth Patterns in the Developing Human Brain Detected Using Continuum-Mechanical Tensor Mapping. Nature, 404:190-193.

[49] Thompson, P.M., Mega, M.S., Woods, R.P., Zoumalan, C.I., Lindshield, C.J., Blanton, R.E., Moussai, J., Holmes, C.J., Cummings, J.L., and Toga, A.W. (2001). Cortical Change in Alzheimer's Disease Detected with a Disease-Specific Population-Based Brain Atlas. Cerebral Cortex, 11:1-16, 2001.

[50] Vasken, K. (1996). Performance Analysis of Automatic Techniques for Tissue Classification in Magnetic Resonance Images of the Human Brain. Master's Thesis, Computer Science, Concordia University, Canada. 1996.

[51] Worsley, K.J., Andermann, M., Koulis, T., MacDonald, D., and Evans., A.C. (1999). Detecting Changes in Non-isotropic Images. Human Brain Mapping, 8:98-101. 
[52] Worsley, K.J., Marrett, S., Neelin, P., Vandal, A.C., Friston, K.J., and Evans, A.C (1996a). A Unified Statistical Approach for Determining Significant Signals in Images of Cerebral Activation. Human Brain Mapping, 4:58-73.

[53] Worsley, K.J., Marrett, S., Neelin, P., and Evans, A.C. (1996b). Searching Scale Space for Activation in Pet Images. Human Brain Mapping, 4:74-90.

[54] Zijdenbos, A.P., Jimenez, A., and Evans, A.C. (1998). Pipelines: Large Scale Automatic Analysis of 3D Brain Data Sets. NeuroImage, 7S:783. 